\title{
Role of hemibonding in the structure and ultraviolet spectroscopy of the aqueous hydroxyl radical
}

\author{
Bhaskar Rana and John M. Herbert* \\ Department of Chemistry and Biochemistry, The Ohio State University, Columbus, Ohio 43210 USA
}

(Dated: October 3, 2020)

\begin{abstract}
The presence of a hemibond in the local solvation structure of the aqueous hydroxyl radical has long been debated, as its appearance in ab initio simulations based on density functional theory is sensitive to self-interaction error (favoring a two-center, three-electron hemibond) but also to finite-size effects. Simulations reported here use a mixed quantum mechanics/molecular mechanics (QM/MM) framework in a very large periodic simulation cell, in order to avoid finite-size artifacts and to facilitate testing of various density functionals, in order to probe the effects of delocalization error. The preponderance of hemibonded structures predicted by generalized gradient approximations persists in simulations using the hybrid functionals B3LYP and PBE0, but is reduced to a minor population if the fraction of exact exchange is increased significantly. The hemibonded population is also small in simulations employing the long-range corrected functional LRC- $\omega$ PBE. Electronic spectra are computed using time-dependent density functional theory, and from these calculations emerges a consensus picture in which hemibonded configurations play an outsized role in the absorption spectrum, even when present as a minority species. An intense $1 b_{2}\left(\mathrm{H}_{2} \mathrm{O}\right) \rightarrow 2 p \pi(\cdot \mathrm{OH})$ charge-transfer transition in hemibonded configuration of the radical proves to be responsible for an absorption feature at $230 \mathrm{~nm}$ that is strongly shifted with respect to the gas-phase absorption at $307 \mathrm{~nm}$, but this intense feature is substantially diminished in aqueous geometries where the hemibond is absent. Although not yet sufficient to quantitatively establish the population of hemibonded $\cdot \mathrm{OH}(\mathrm{aq})$, these simulations do suggest that its presence is revealed by the strongly shifted ultraviolet absorption spectrum of the aqueous radical.
\end{abstract}

\section{Introduction}

The hydrated hydroxyl radical plays a significant role in biological, atmospheric and industrial processes. 115 The solvation structure of $\cdot \mathrm{OH}(\mathrm{aq})$ has long been studied via $a b$ initio molecular dynamics $\left[\frac{622}{22}\right.$ Its diffusion constant is nearly identical to water's self-diffusion constant (see Table 1,, 23124 and is therefore much slower than Grotuß-assisted diffusion of either $\mathrm{H}^{+}(\mathrm{aq})$ or $\mathrm{OH}^{-}(\mathrm{aq}), 25$ 27 or the librationally-assisted diffusion of $e^{-}(\mathrm{aq}) .28$ What has proved most contentious, however, is the putative existence of a two-center, three-electron "hemibond" in the first solvation shell of $\cdot \mathrm{OH}(\mathrm{aq})$. An example of such a structure is shown in Fig. 1 for the complex of $\cdot \mathrm{OH}$ with a single water molecule. ${ }^{\frac{29}{29}}$ The hemibonded motif is available due to a favorable overlap between the singly-occupied $2 p \pi$ molecular orbital (MO) of the radical and the frontier $1 b_{2}$ lone pair of a neighboring water molecule. $\frac{15}{15}$

That said, it was considered early on that this delocalized sharing of the unpaired electron might be artificially stabilized by self-interaction error (SIE) in density-functional calculations. ${ }^{6]} 8$ Over-delocalization of radical centers is a general problem in density functional theory (DFT), $\stackrel{3133}{33}$ e.g., for the description of polarons in $\mathrm{TiO}_{2}, \frac{34}{3}$ or when hole defects are intro-

*herbert@chemistry.ohio-state.edu
Table 1: Diffusion constants for some aqueous species at $25^{\circ} \mathrm{C}$.

\begin{tabular}{llc}
\hline \hline Species & $D\left(\AA^{2} / \mathrm{ps}\right)$ & Ref. \\
\hline $\mathrm{H}^{+}$ & 0.93 & 27 \\
$\mathrm{H}^{\bullet}$ & $0.7-0.8$ & 23 \\
$\mathrm{OH}^{-}$ & 0.53 & 27 \\
$e^{-}$ & $0.48-0.49$ & 23 \\
$\mathrm{H}_{2} \mathrm{O}$ & 0.23 & 30 \\
$\mathrm{HO}^{\bullet}$ & $0.21-0.23$ & 23 \\
$\mathrm{Cl}^{-}$ & 0.20 & 27 \\
\hline \hline
\end{tabular}

duced into silica $\sqrt[35-37]{3}$ in cation radicals such as ionized

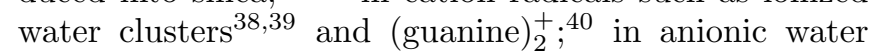
clusters $\stackrel{41}{43}$ in radical-molecule complexes, $\stackrel{4445}{, 4 n c l u d-}$ ing complexes of $\mathrm{H}_{2} \mathrm{O}$ with small open-shell atoms and ions $\stackrel{44}{, 4}$ and in ion-radical complexes such as $\mathrm{HOCl}^{-} \underline{46 / 47}$ In the latter case, SIE overstabilizes the hemibonded configuration $\mathrm{HO} \cdot \cdots \mathrm{Cl}^{-}$, relative to the hydrogen-bonded geometry $\cdot \mathrm{OH} \cdots \mathrm{Cl}^{-} \stackrel{46 / 47}{ } \mathrm{In}$ the case of $\cdot \mathrm{OH}(\mathrm{aq})$, it was considered that the appearance of hemibonded configurations in $a b$ initio molecular dynamics (aiMD) simulations might therefore be an artifact of SIE, 6 , 8 although more recently this viewpoint has been challenged by Kusalik and co-workers, $\frac{12}{15}$ as discussed below.

A summary of previous aiMD simulations of $\cdot \mathrm{OH}(\mathrm{aq})$ is presented in Table 2 In some of these studies, the presence of a hemibonded solvation motif manifests as a distinct feature in the $\mathrm{O}^{*} \cdots \mathrm{O}$ radial distribution function $(\mathrm{RDF})$, where $\mathrm{O}^{*}$ denotes the oxygen atom of the radical. This feature, if present, appears around $r_{\mathrm{O}^{*} \mathrm{O}} \approx 2.3-$ 
Table 2: Summary of previous ai MD studies of $\bullet \mathrm{OH}(\mathrm{aq})$, indicating whether or not the authors report a hemibonded solvation structure.

\begin{tabular}{|c|c|c|c|c|}
\hline Reference & Unit Cell & Functional & Hemibond? & $T(\mathrm{~K})$ \\
\hline Khalack and Lyubartsev $\sqrt{9}(2004)$ & $\cdot \mathrm{OH}\left(\mathrm{H}_{2} \mathrm{O}\right)_{31}$ & BLYP & $\sqrt{ }$ & 310 \\
\hline Vassilev et $a l^{[6]}(2005)$ & $\cdot \mathrm{OH}\left(\mathrm{H}_{2} \mathrm{O}\right)_{31}$ & BLYP & $\checkmark$ & 300 \\
\hline VandeVondele and Sprik & $\cdot \mathrm{OH}\left(\mathrm{H}_{2} \mathrm{O}\right)_{31}$ & BLYP-SIC & $x$ & 350 \\
\hline Codorniu-Hernandez and Kusalik ${ }^{[12}$ (2011) & $\mid \begin{array}{l}\cdot \mathrm{OH}\left(\mathrm{H}_{2} \mathrm{O}\right)_{31} \\
\cdot \mathrm{OH}\left(\mathrm{H}_{2} \mathrm{O}\right)_{31} \\
\cdot-\mathrm{OH}\left(\mathrm{H}_{2} \mathrm{O}\right)_{63} \\
\cdot \cdot \mathrm{OH}\left(\mathrm{H}_{2} \mathrm{O}\right)_{63}\end{array}$ & $\begin{array}{l}\text { BLYP } \\
\text { HCTH/120 } \\
\text { BLYP } \\
\text { HCTH/120 }\end{array}$ & $\begin{array}{l}\checkmark \\
\checkmark \\
x \\
x\end{array}$ & $\begin{array}{l}310 \\
310 \\
310 \\
310\end{array}$ \\
\hline Genova et al. $\frac{19}{(2016)}$ & $\begin{array}{l}\cdot \mathrm{OH}\left(\mathrm{H}_{2} \mathrm{O}\right)_{63} \\
\cdot \mathrm{OH}\left(\mathrm{H}_{2} \mathrm{O}\right)_{63}\end{array}$ & $\begin{array}{l}\text { revAPBEK } \\
\text { revAPBEK-SIC }\end{array}$ & $\begin{array}{l}x \\
x\end{array}$ & $\begin{array}{l}340 \\
340\end{array}$ \\
\hline von Rudorff et al. ${ }^{[20}$ (2017) & $\begin{array}{l}\cdot \mathrm{OH}\left(\mathrm{H}_{2} \mathrm{O}\right)_{31} \\
\cdot \mathrm{OH}\left(\mathrm{H}_{2} \mathrm{O}\right)_{31}\end{array}$ & $\begin{array}{l}\text { BLYP+D3 } \\
\text { HSE06+D3 }\end{array}$ & $\begin{array}{l}\checkmark \\
x\end{array}$ & - \\
\hline Apostolidod ${ }^{21}(2019)$ & $\begin{array}{l}\cdot \mathrm{OH}\left(\mathrm{H}_{2} \mathrm{O}\right)_{31} \\
\cdot \mathrm{OH}\left(\mathrm{H}_{2} \mathrm{O}\right)_{31} \\
\cdot \mathrm{OH}\left(\mathrm{H}_{2} \mathrm{O}\right)_{31}\end{array}$ & $\begin{array}{l}\text { B3LYP+D3 } \\
\text { PBE0+D3 } \\
\text { HSE03+D3 }\end{array}$ & $\begin{array}{l}x \\
x \\
x\end{array}$ & $\begin{array}{l}370 \\
370 \\
370\end{array}$ \\
\hline
\end{tabular}

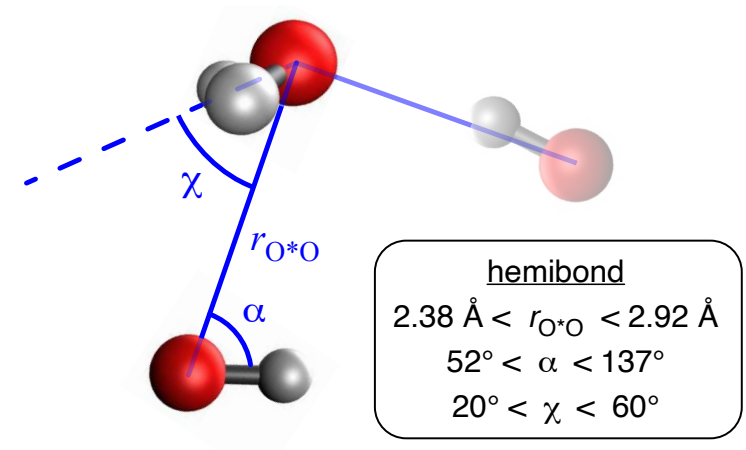

Figure 1: $\quad$ Schematic view of the hemibonded $\mathrm{H}_{2} \mathrm{O} \cdots \cdot \mathrm{OH}$ complex (opaque models) along with a translucent hydroxyl radical in a hydrogen-bonded orientation. Quantitative suggestions for the geometrical parameters that define the hemibonded structure are taken from Ref. 29.

$2.4 \AA$ A. Vassilev et al ${ }^{[67}$ and Khalack et al.$^{[9}$ performed $a i \mathrm{MD}$ simulations using the BLYP functional, $48 / 49$ placing 31 waters (along with $\cdot \mathrm{OH}$ ) in the simulation cell. A peak in the aforementioned RDF around $r_{\mathrm{O}^{*} \mathrm{O}} \approx 2.3 \AA$ was observed, and explained as the presence of the hemibond as the average coordinate structure, though it was acknowledged at the time that this could be an artifact of SIE ${ }^{[6}$ Recognizing this, VandeVondele and Sprik added an empirically-parameterized self-interaction correction (SIC) to BLYP, and observed that the hemibonded structures disappeared in simulations that employed this BLYP-SIC functional. For a time, this study cemented the conventional wisdom that the hemibonded structure is an artifact of SIE.

However, this interpretation was subsequently challenged by Codorniu-Hernández and Kusalik.12 $[15]$ These authors do observe a hemibonded structure when $\cdot \mathrm{OH}(\mathrm{aq})$ is simulated in a periodic cell with $N=31$ water molecules, using either the BLYP and $\mathrm{HCTH} /$ $120^{\sqrt[50]{0}}$ functionals, with no SIC!12 When the periodic cell is expanded to $\cdot \mathrm{OH}\left(\mathrm{H}_{2} \mathrm{O}\right)_{63}$, however, the hemibonded configurations disappear. Later, these authors computed potentials of mean force along the $\mathrm{O}^{*} \ldots \mathrm{O}$ coordinate using various functionals (in the larger box), and demonstrated the existence of the hemibond as an alternative structure in these simulations, with a barrier of $2-3 \mathrm{kcal} / \mathrm{mol}$ separating it from the hydrogen-bonded structure $!^{15}$ Although Codorniu-Hernández et al ${ }^{12115}$ attribute the hemibonded solvation motif to an artifact of finite simulation size, this does not entirely explain its disappearance, in smaller simulations using $N=31$ water molecules, when an SIC is added to BLYP 8 In the larger $(N=63)$ simulation cell, aiMD simulations suggest that the hemibonded structure is absent whether a SIC is applied or not.19

The phenomenon of SIE is often associated with semilocal functionals developed within the generalized gradient approximations (GGA), and hybrid functionals that introduce a fraction of "exact" (Hartree-Fock) exchange are viewed as an antidote to this problem. Especially in the condensed-matter simulation community, we observe a tendency to view hybrid functionals such as PBE0, $[51$ with $25 \%$ exact exchange, as being largely free of the effects of SIE, although this is not true and does not stand up to close scrutiny. ${ }^{52 \mid 53}$ Often, hybrids containing $\gtrsim 50 \%$ exact exchange being needed to substantially eliminate SIEs in barrier heights,,$\sqrt[5455]{55}$ with deleterious effects on other predicted properties $[54$ It is true, however, that the (de)localization of an unpaired spin may correlate with the fraction of exact exchange $e^{35 / 37 / 40}+42$ Application of a SIC to the singly-occupied MO (SOMO) also serves to localize the unpaired spin. $\underline{40 \mid 43}$

Hybrid-functional aiMD simulations have recently been brought to bear on $\cdot \mathrm{OH}(\mathrm{aq})$ by Apostolidou, ${ }^{21}$ using the smaller $(N=31)$ simulation cell in conjunction 
with the B3LYP $49[56$ PBE0,51 and HSE0357 functionals. (Unlike other simulations mentioned above, these ones also employed an empirical dispersion correction, + D3 ${ }^{58}$ ) In these simulations, the average structure is hydrogen-bonded rather than hemibonded, despite the use of a smaller cell, suggesting perhaps a delicate interplay between simulation size, SIE, and the structure that is obtained. In the present work, we will use a quantum mechanics/molecular mechanics (QM/MM) framework that facilitates the use of a very large periodic cell, in order to avoid any potential artifacts due to finite-size effects.

Apart from these questions regarding the structure of $\cdot \mathrm{OH}(\mathrm{aq})$, there has been separate experimental and theoretical efforts to assign the peak in the ultraviolet absorption spectrum of this species, and hemibonded configurations have been suggested to play a role in the very intense absorption of this species. ${ }^{29}$ In the gas phase, $\cdot \mathrm{OH}$ absorbs at $307 \mathrm{~nm}(4.04 \mathrm{eV}) \stackrel{[59[60}{,}$ corresponding to the $\tilde{\mathrm{A}}^{2} \Sigma^{+}\left(\sigma_{\mathrm{lp}}^{2} \sigma_{\mathrm{b}}^{1} \pi^{4}\right) \leftarrow \tilde{\mathrm{X}}^{2} \Pi\left(\sigma_{\mathrm{lp}}^{2} \sigma_{\mathrm{b}}^{2} \pi^{3}\right)$ transition. ${ }^{61}$ The absorption maximum of $\cdot \mathrm{OH}(\mathrm{aq})$, however, is shifted to $230 \mathrm{~nm}(5.4 \mathrm{eV}), \frac{62 \sqrt[65]{65}}{6 u c h}$ too large to be a non-specific medium effect (e.g., from the dielectric environment) $\frac{6164}{6}$ although this explanation has been suggested ${ }^{66}$ More recently, the temperature dependence of the $\cdot \mathrm{OH}(\mathrm{aq})$ absorption spectrum was measured, 67 showing that a weaker band at $310 \mathrm{~nm}$ grows in intensity at higher temperature while the stronger band at $230 \mathrm{~nm}$ diminishes. The transition at $230 \mathrm{~nm}$ was ascribed to a charge-transfer (CT) transition from a hydrogen-bonded water molecule to the radical, whereas the band near $310 \mathrm{~nm}$ was assigned to the localized transition on the radical itself, analogous to the gas-phase transition at $307 \mathrm{~nm} 67$

Theoretical studies of the spectroscopy of the hydrated - $\mathrm{OH}$ radical have so far been limited to gas-phase cluster studies. ${ }^{2916168]} 70$ Excited states of the binary $\cdot \mathrm{OH}\left(\mathrm{H}_{2} \mathrm{O}\right)$ complex have been reported using equation-of-motion coupled-cluster (EOM-CC) calculations including connected triples (EOM-CCSDT) ${ }^{29161}$ and small clusters - $\mathrm{OH}\left(\mathrm{H}_{2} \mathrm{O}\right)_{n}$ have also been studied using EOM-CCSD,

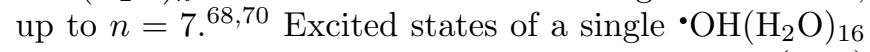
cluster have been reported using time-dependent (TD-) DFT ${ }^{68}$ Each of these studies confirms the existence of a $\mathrm{H}_{2} \mathrm{O} \rightarrow \cdot \mathrm{OH}$ transition whose intensity is sensitive to orbital overlap, $\frac{68}{6}$ and which is suggested to be responsible for the aqueous-phase absorption at $230 \mathrm{~nm}$. Chipman 29 specifically ascribes the intense CT transition to hemibonded configurations of the radical, which he suggests might be rare in the liquid but which nevertheless dominate the absorption spectrum of $\cdot \mathrm{OH}(\mathrm{aq})$ by virtue of a very large oscillator strength. So far, however, there are no condensed-phase simulations of the absorption spectrum that might tie this observable to the aforementioned structural ambiguities surrounding the hemibonded coordination motif. Such studies are reported here for the first time.

We report $\mathrm{QM} / \mathrm{MM}$ simulations of $\cdot \mathrm{OH}(\mathrm{aq})$ using a large, periodic simulation cell and various density functionals ranging from BLYP to hybrids (B3LYP and PBE0) and finally a range-separated hybrid, LRC$\omega \mathrm{PBE}{ }^{71}$ Hemibonded configurations appear in each of these simulations, to a greater or lesser extent that we will analyze. Ensemble-averaged absorption spectra are then computed using TD-DFT in an effort to test the aforementioned hypothesis, that hemibonded configurations play an outsized role in the absorption spectrum of $\cdot \mathrm{OH}(\mathrm{aq})$.

\section{Computational Details}

\subsection{QM/MM Simulations}

All QM/MM simulations were performed using QChem, ${ }^{72}$ v. 5.1. The $\mathrm{QM}$ region was described using either B3LYP, PBE0, or LRC- $\omega$ PBE in conjunction with the $6-31 \mathrm{G}^{*}$ basis set and the D3 dispersion correction.58 A range-separation parameter $\omega=0.380 \mathrm{bohr}^{-1}$ is used for LRC- $\omega \mathrm{PBE}$, which is very close to the "tuned" values that are selected below based on the ionization energy theorem ${ }^{[3]}$ Trajectories were initiated from an equilibrated liquid water trajectory by removing one hydrogen atom near the center of the simulation cell and then performing energy minimization prior to MD simulations.

Periodic boundary conditions were applied using a cubic box of length $L=31.342 \AA$, containing 1,023 water molecules and one hydroxyl radical. This affords a density of $0.997 \mathrm{~g} / \mathrm{cm}^{3}$, matching liquid water's density at $T=300 \mathrm{~K}$. The $\mathrm{QM}$ region was selected to match the smaller simulation cells used in previous aiMD simulations (see Table 2), taking the $N=31$ water molecules that lie within a radius of $5.8 \AA$ of the radical. The remaining water molecules are described using the TIP3P force field ${ }^{74}$ All simulations were performed under constant $N V T$ conditions using a Nosé-Hoover thermostat ${ }^{75}$ set to $T=300 \mathrm{~K}$. Initial velocities were sampled from a Maxwell-Boltzmann velocity distribution at $T=300 \mathrm{~K}$ and then propagated using the velocity Verlet algorithm with a time step of 42 a.u. $(=1.016 \mathrm{fs})$. The selfconsistent field (SCF) guess was regenerated at each time step in order to avoid energy drift caused by violation of time-reversal symmetry ${ }^{[76}$ The SCF convergence threshold was set to $\tau_{\mathrm{SCF}}=10^{-6} E_{h}$, sufficient for energy conservation. ${ }^{76}$

Periodic boundary conditions were implemented using the QM/MM-Ewald technique that we have previously described ${ }^{77 / 78}$ and applied to systems such as $e^{-}(\mathrm{aq}){ }^{78 / 79}$ Periodic images of the QM atoms are represented using ChEIPG charges, ${ }^{80}{ }^{82}$ derived from the QM electrostatic potential. For efficiency, we use an implementation of the ChElPG algorithm based on atom-centered Lebedev grids, ${ }^{77}$ with 50 angular points per radial shell. These shells begin at the atomic van der Waals radius of each atom and extend for another $5 \AA$, in $0.3 \AA$ intervals. Sep- 
arate Ewald splitting parameters are used for to describe the $\mathrm{MM} / \mathrm{MM}$ and QM/MM interactions. Following previous work,, $77 / 78$ the Ewald splitting parameter $\eta_{\mathrm{MM}}$ for the $\mathrm{MM} / \mathrm{MM}$ interactions is taken to be $\eta_{\mathrm{MM}}=2 C / L$ where

$$
C=\left[-\ln \left(\tau_{\mathrm{SCF}} / E_{h}\right)\right]^{1 / 2} .
$$

With $\tau_{\mathrm{SCF}}=10^{-6} E_{h}$, this affords $\eta_{\mathrm{MM}}=0.237 \AA^{-1}$. An optimal splitting parameter $\eta_{\mathrm{QM}}$ for the $\mathrm{QM} / \mathrm{MM}$ interactions is estimated using the equation ${ }^{77 / 78}$

$$
\frac{2 C L^{3} \eta_{\mathrm{QM}}^{3}}{\pi^{3 / 2}}+\frac{L^{2} \eta_{\mathrm{QM}}^{2}}{\pi^{1 / 2}}-L \eta_{\mathrm{QM}}-2 C=0
$$

from which we obtain $\eta_{\mathrm{QM}}=0.0566 \AA^{-1}$.

We simulate 5 ps trajectories but exclude from the analysis the first $0.5 \mathrm{ps}$, during which the temperature equilibrates (see Fig. S1). We also exclude any portion of the trajectory where the radical approaches the QM/ MM boundary, or where there is significant penetration of MM water molecules into the QM region. In principle, this could be avoided through the use of adaptivepartitioning QM/MM boundary conditions, ${ }^{83}$ but at the cost of greatly increasing the complexity and cost of the simulation. Alternatively a confining potential could be added to the QM/MM interface, $\frac{84}{\text { at }}$ the risk of introducing artifacts in the local solvation structure. We have chosen not to apply these techniques, which limits our trajectories to $4-5$ ps in duration.

\subsection{Absorption Spectrum}

Absorption spectra were computed by extracting $80-$ 100 snapshots from each trajectory, separated by 50 fs intervals. From each snapshot, a TD-DFT/MM calculation was prepared in the following way. First, a $3 \times 3$ supercell was formed from the periodic simulation cell, and from that supercell a QM region extending to a radius of $5.5 \AA$ around the $\cdot \mathrm{OH}$ was selected, containing 30-34 water molecules. The remaining atoms in the supercell were represented by MM point charges. TD-DFT calculations were performed within the Tamm-Dancoff approximation. 85

At each snapshot, we first compute a vertical excitation spectrum consisting of the lowest 15 excited states, whose oscillator strengths are given by

$$
f_{0 n}=\left(\frac{2 m_{e} \omega_{0 n}}{3 \hbar}\right) \sum_{\kappa=x, y, z}\left|\left\langle\Psi_{0}|\hat{\kappa}| \Psi_{n}\right\rangle\right|^{2}
$$

where $\omega_{0 n}=\left(E_{n}-E_{0}\right) / \hbar$. We then apply Lorentzian broadening (with a width of $0.2 \mathrm{eV}$ ) to each vertical transition, and weight each transition by its oscillator strength $f_{0 n}$. Finally, an ensemble-averaged complete spectrum is obtained by averaging these individual broadened spectra.
Four different functionals are tested for the TD-DFT calculations, in conjunction with the $6-31++\mathrm{G}^{*}$ basis set. These include the BH\&HLYP functional, which Chipman found to afford satisfactory agreement with EOM-CCSDT benchmarks for the $\cdot \mathrm{OH}\left(\mathrm{H}_{2} \mathrm{O}\right)$ complex, 61 and also CAM-B3LYP ${ }^{86}$ Anticipating an important CT transition, we have used two long-range corrected (LRC) functionals, namely, LRC- $\omega \mathrm{PBE}^{71}$ and LRC$\mu \mathrm{BLYP}, 8788$ each of which exhibits proper asymptotic behavior for a well-separated separated electron and hole. Range-separation parameters $(\omega$ or $\mu)$ were tuned separately for each of the trajectories, using the "optimal tuning" (OT) procedure ${ }^{73}$ in an ensemble-averaged way. Specifically, we use 8-10 well-separated snapshots to find the value of $\omega$ that best satisfies the condition

$$
\mathrm{IE}(\omega)=-\varepsilon_{\mathrm{SOMO}}(\omega)
$$

in an average way for all snapshots. These tuned functionals are denoted OT-LRC- $\omega$ PBE and OT-LRC$\mu \mathrm{BLYP}$.

\section{$3 \quad$ Structure of $\cdot \mathrm{OH}(\mathrm{aq})$}

We will divide the discussion of our results into two parts. In this section, we discuss structural aspects of the aqueous hydroxyl radical and compare to previous work. The absorption spectroscopy, which is carried out for the aqueous phase for the first time, is discussed in Section 4, where we connect the spectroscopy back to the structural motifs (hemibond versus hydrogen bond) that are discussed here. Throughout this discussion, the atoms of the hydroxyl radical are indicated as $\mathrm{O}^{*}$ and $\mathrm{H}^{*}$.

\subsection{Radial distribution functions}

Figure 2 compares the RDFs $g\left(r_{\mathrm{O}^{*} \mathrm{O}}\right)$ from the various simulations. These are computed using a bin width of $0.05 \AA$ using the snapshots from the post-equilibration period. As discussed in previous work, $\frac{6|8| 9|12| 19}{21}$ this particular RDF plays an important role in identifying the presence or absence of a hemibonded configuration, with a sharp peak around $r_{\mathrm{O}^{*} \mathrm{O}} \approx 2.3-2.4 \AA$ indicating that the hemibond exists as the predominant structure rather than as a rare event or fluctuation. ${ }^{8}$ In the B3LYP +D3 and PBE0+D3 simulations reported here, this peak appears to be present but is not nearly so pronounced as it is in previous studies that report a hemibonded structure. Especially in the case of our PBE0 simulations, the hemibonded feature appears as a shoulder that is partially subsumed into a larger peak centered at $r_{\mathrm{O}^{*} \mathrm{O}} \approx 2.6-2.7 \AA$ that represents the ever-present $\mathrm{HO} \cdot \cdots \mathrm{H}_{2} \mathrm{O}$ hydrogen bonds.

In the LRC- $\omega \mathrm{PBE}+\mathrm{D} 3$ simulation, however, there is no evidence for a distinct feature in the RDF that might 


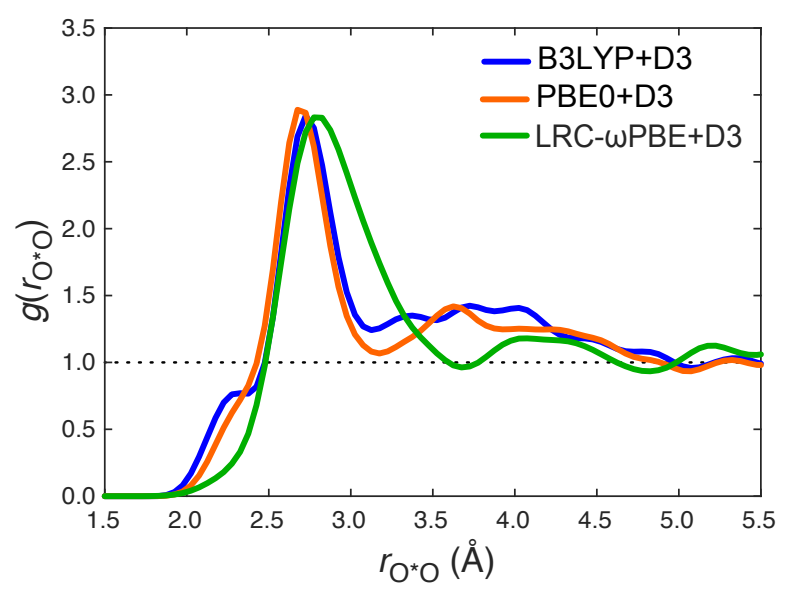

Figure 2: Comparison of RDFs $g\left(r_{\mathrm{O} * \mathrm{O}}\right)$ for $\bullet \mathrm{OH}(\mathrm{aq})$, where $\mathrm{O}^{*}$ indicates the hydroxyl oxygen. Results were obtained from $\mathrm{QM} / \mathrm{MM}$ simulations using three different density functionals.

correspond to a hemibond. It is possible that it could be obscured by the hydrogen-bonding peak, which is much broader in the case of LRC- $\omega \mathrm{PBE}+\mathrm{D} 3$ than it is for the other two functionals, indicating a less structured liquid. Based on calculations for binary $\mathrm{HO} \cdots \mathrm{H}_{2} \mathrm{O}$ complexes, Chipman ${ }^{29}$ has suggested that the hemibond could be characterized by a broad distribution of $\mathrm{O}^{*} \ldots \mathrm{O}$ distances, roughly $r_{\mathrm{O}^{*} \mathrm{O}} \approx 2.38-2.92 \AA$ as indicated in Fig. 1] If a hemibonded configuration does exist in the $\mathrm{LRC}-\omega \mathrm{PBE}+\mathrm{D} 3$ simulations but is characterized by a slightly larger value of $r_{\mathrm{O}^{*} \mathrm{O}}$ than in other simulations, it is possible that this feature could hide underneath the larger distribution of hydrogen-bonded $\mathrm{O}^{*} \ldots \mathrm{O}$ distances. Below, we will introduce definitions of the hemibond that are based on just on geometrical structure but also on examination of the spin density, which suggest that hemibonded configurations do exist in the LRC$\omega \mathrm{PBE}+\mathrm{D} 3$ simulation although they are a minority population.

To investigate the extent to which the fraction of exact exchange plays a role in the presence or absence of this hemibonded shoulder in $g\left(r_{\mathrm{O}^{*} \mathrm{O}}\right)$, we have also run few shorter trajectories with the BLYP+D3 and BH\&HLYP + D3 functionals, and with dispersion-corrected HartreeFock theory $(\mathrm{HF}+\mathrm{D} 3)$. The resulting $\mathrm{O}^{*} \ldots \mathrm{O}$ RDFs are provided in Fig. S3 of the Supporting Information. For BLYP $+\mathrm{D} 3$, the hemibonded peak at $r_{\mathrm{O}^{*} \mathrm{O}} \approx 2.3 \AA$ is much more pronounced than it is even in the B3LYP + D3 simulation (Fig. $\mathrm{S3}$ ) and is consistent with the BLYP results reported by VandeVondele and Sprik prior to application of the SIC ${ }^{8}$ In contrast, simulations with BH\&HLYP+D3 (with 50\% exact exchange) and HF+D3 afford RDFs that are very similar to those obtained with LRC- $\omega$ PBE+D3; see Fig. S3b. Together, these comparisons hint that the hemibonded feature observed in our B3LYP +D3 and PBE0+D3 simulations may stem from SIE, despite the fact that these are hybrid functionals.

It is also worth noting that our B3LYP+D3 and PBE0
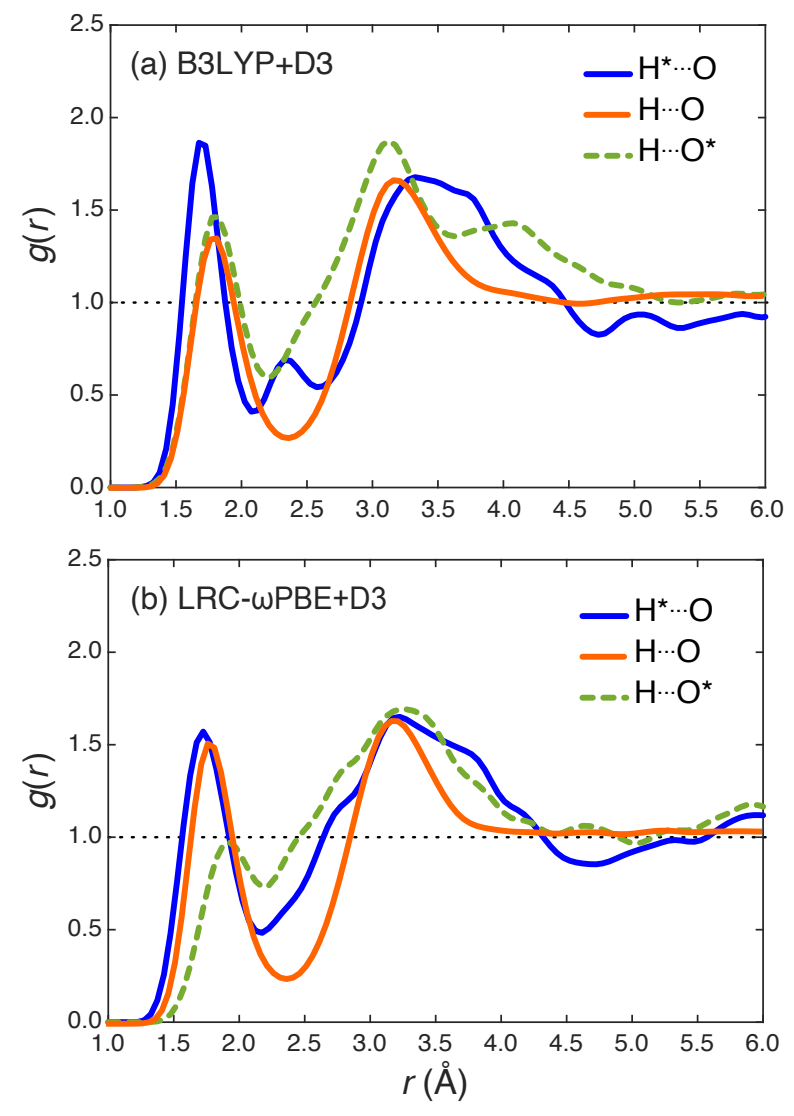

Figure 3: Comparison of different oxygen-hydrogen RDFs obtained from (a) B3LYP + D3 versus (b) LRC- $\omega$ PBE + D3 simulations. Covalent bonds have been excluded from the averaging so that $g\left(r_{\mathrm{OH}}\right)$ represents the RDF for hydrogen bonds only.

$+\mathrm{D} 3$ results disagree with recent aiMD simulations that were reported using the same functionals, $\stackrel{21}{21}$ in which no feature at small $r_{\mathrm{O}^{*} \mathrm{O}}$ is observed. Simulations reported in Ref. 21 used a periodic cell containing $N=31$ water molecules, and may therefore be subject to finitesize artifacts. If so, then it is notable that such artifacts induce the formation of a hemibonded structure when GGA functionals are employed (as documented in Ref. 12), but disrupt the hemibond when hybrid functionals are employed. We have no further explanation for these discrepancies except to note that it is clear from our own simulations (Fig. 2) that the distribution of hydrogen bond lengths is sensitive to the choice of functional and that depending on the details of this distribution, the hydrogen-bonding peak in $g\left(r_{\mathrm{O}^{*} \mathrm{O}}\right)$ may obscure the hemibonded peak, partially if not fully. Indeed, we will document below that hemibonded structures are present even in the LRC- $\omega \mathrm{PBE}+\mathrm{D} 3$ simulations that we report (although they are a minority population), despite the absence of any distinct feature in $g\left(r_{\mathrm{O}^{*} \mathrm{O}}\right)$ that would indicate their presence.

The comparison among the oxygen-hydrogen RDFs is 
also important, specifically to characterize the strength of $\bullet \mathrm{OH}$ as a hydrogen-bond donor or acceptor. Figure 3 compares the three different types of oxygen-hydrogen RDF: $\mathrm{H} \cdots \mathrm{O}^{*}, \mathrm{H}^{*} \cdots \mathrm{O}$, and $\mathrm{H} \cdots \mathrm{O}$. (The PBE0+D3 results are similar to the B3LYP + D3 results and can be found in Fig. S4) As compared to the $\mathrm{H} \cdots \mathrm{O}$ distribution, which measures the distribution of water-water hydrogen bond lengths, the first peak in the $\mathrm{H}^{*} \ldots \mathrm{O}$ distribution appears at a shorter distance, suggesting that the $\cdot \mathrm{OH}$ is a better hydrogen-bond donor as compared to a water molecule. However, the strength of $\bullet \mathrm{OH}$ as a hydrogen-bond acceptor differs in the two simulations shown in Fig. 3, as becomes apparent in comparing the $\mathrm{H} \cdots \mathrm{O}^{*}$ and $\mathrm{H} \cdots \mathrm{O}$ distributions. According to B3LYP +D3 results, hydroxyl is a comparable hydrogen-bond acceptor as compared to water, as the first solvationshell structure of $g\left(r_{\mathrm{HO}^{*}}\right)$ is almost identical to that of $g\left(r_{\mathrm{HO}}\right)$. In contrast, for the LRC- $\omega \mathrm{PBE}+\mathrm{D} 3$ simulation, - $\mathrm{OH}$ is slightly less active as a hydrogen-bond acceptor (in comparison to a water molecule), as here the first maximum in $g\left(r_{\mathrm{HO}^{*}}\right)$ is peaked at a larger value of $r$ and is much less intense as compared to $g\left(r_{\mathrm{HO}}\right)$. Comparing the two simulations, one can say that the distribution of $\mathrm{O}-\mathrm{H} \cdots \mathrm{O}^{*}$ hydrogen bonds is much less structured in the LRC- $\omega$ PBE + D3 simulation.

It is worth noting that the more structured B3LYP $+\mathrm{D} 3$ results presented here for oxygen-hydrogen RDFs are generally in agreement with previous aiMD simulations using the GGA functional BLYP in small simulation cells ${ }^{[89}$ In contrast, BLYP-SIC result $s^{88}$ more closely resemble our LRC- $\omega \mathrm{PBE}+\mathrm{D} 3$ results. As noted above, this suggests a very subtle interplay between simulation size, self-interaction, and the fraction of exact exchange.

\subsection{Counting hydrogen bonds}

To further investigate the local solvation structure and hydrogen-bonding network around the radical, we quantify the number of water...hydroxyl hydrogen bonds at each time step by taking the nearest five water molecules to the $\cdot \mathrm{OH}$ and using an angle-dependent distance cutoff,, 89190

$$
r_{\text {cut }}(\theta) / \AA=-0.00044(\theta / \mathrm{deg})^{2}+3.3,
$$

to determine whether a hydrogen bond exists or not. Here, $\theta$ is the angle between the $\mathbf{r}_{\mathrm{OO}}$ and $\mathbf{r}_{\mathrm{OH}}$ vectors. For angles $\theta \leq 50^{\circ}$, we judge that a hydrogen bond exists if $r_{\mathrm{O}^{*} \mathrm{O}} \leq r_{\text {cut }}$. Histograms of the number of hydrogen bonds determined in this way are reported in Fig. 4. Fitted probability distributions are superimposed on each histogram. (Results for the three functionals are superimposed on one another in Fig. S9.)

For both the B3LYP+D3 and PBE0+D3 simulations, the average number of hydrogen bonds in the first solvation shell is $\langle n\rangle=3$, which is also the most probable number. Of these three hydrogen bonds, the one where the radical acts as the hydrogen donor is very stable during the dynamics. In the other two-more fluxionalhydrogen bonds, the $\mathrm{O}^{*}$ acts as the acceptor. There is also a fourth water molecule that forms a hemibond with the $\cdot \mathrm{OH}$ for more than half of the snapshots. To check whether this seemingly itinerant fourth water molecule might be an artifact of the cutoff criterion used in Eq. (5), we increased the cutoff from 3.3 to $3.4 \AA$. This modification alters the (already small) number of structures with $n=4$ hydrogen bonds by $<2 \%$, suggesting that the distributions in Fig. 4 are reasonably stable with respect to the cutoff criterion.

The LRC- $\omega$ PBE + D3 results are somewhat different, with $n \approx 2$ hydrogen bonds on average but a broader distribution as compared to those observed using the global hybrid functionals. The $\mathrm{H}^{*}$-donor hydrogen bond persists in simulations with the range-separated hybrid, so the difference is approximately one fewer hydrogen bonds in which $\mathrm{O}^{*}$ acts as an acceptor. Because the first solvation shell of $\cdot \mathrm{OH}$ contains $\approx 5$ water molecules, this means that two of these molecules remain uncoordinated to the radical, on average, although we observe intermittent structures where one of these forms a hemibond with the radical. Consistent with the RDFs discussed above, this counting of the hydrogen bonds suggests that the local solvation environment around $\bullet \mathrm{OH}$ is less structured in the case of LRC- $\omega \mathrm{PBE}+\mathrm{D} 3$ than it is for either PBE0 $+\mathrm{D} 3$ or B3LYP $+\mathrm{D} 3$ and in fact the former closely resembles results obtained by VandeVondele and Sprik using the BLYP-SIC functional ${ }^{[8]}$ In contrast, the distribution of hydrogen bonds obtained from the two global hybrid functionals closely matches with previously-reported BLYP results. 6 [

\subsection{Hemibond formation}

Given the differences in local solvation structure obtained using different functionals, we next examine some correlated joint probability distributions between geometric parameters, in an effort to provide some range of parameters that might characterize hemibonded structures. Chipman ${ }^{29}$ has previously offered such suggestions, which are given in Fig. 1 . but these were based on calculations for the binary $\mathrm{HO} \cdots \mathrm{H}_{2} \mathrm{O}$ complex and may not be fully transferable to the condensed phase. In Figs. 5 and 6, we examine correlations between the geometrical parameters $r_{\mathrm{O}^{*} \mathrm{O}}, \alpha$, and $\chi$ (as defined in Fig. 1), in the form of two-dimensional joint probability distributions. (We use the water molecule closest to the radical in order to define these parameters.) The distributions in Figs. 5 and 6 are based on 400-500 snapshots separated in time by $10 \mathrm{fs}$ intervals.

Two-dimensional distributions in $r_{\mathrm{O}^{*} \mathrm{O}}$ and $\alpha$ are shown in Fig. 5 A bimodal probability distribution is obtained for each of the three functionals that we examine in detail, which is similar for the B3LYP+D3 and PBE0 + D3 functionals but somewhat different for LRC- $\omega$ PBE 

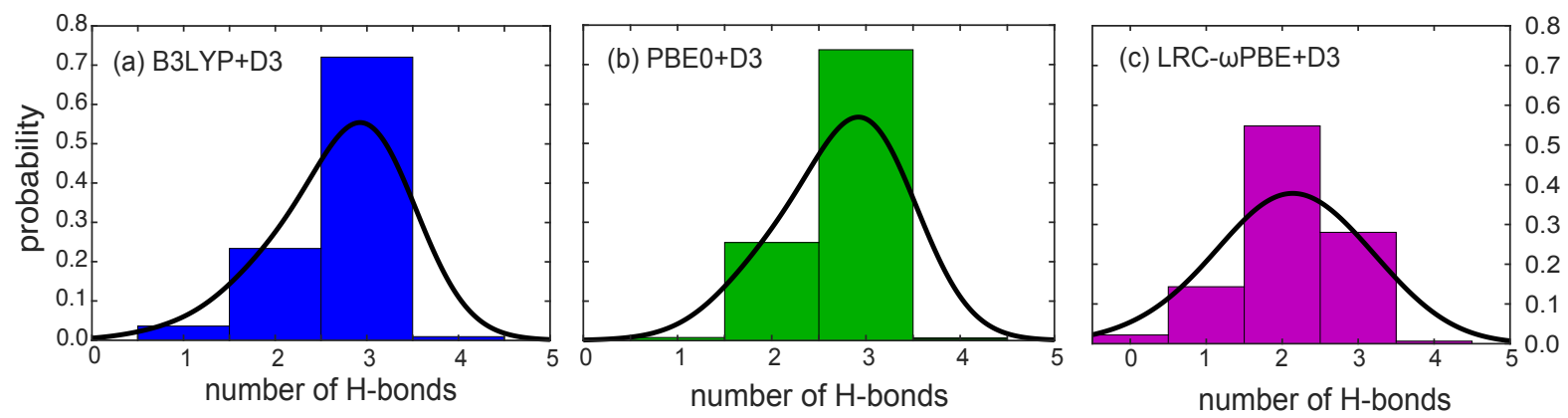

Figure 4: Histograms for the number of hydrogen bonds to $\bullet \mathrm{OH}$, considering the nearest five water molecules to the radical, for simulations performed using (a) B3LYP+D3, (b) PBE0+D3, and (c) LRC- $\omega$ PBE+D3. Black curves are fits to the normalized probability distributions.
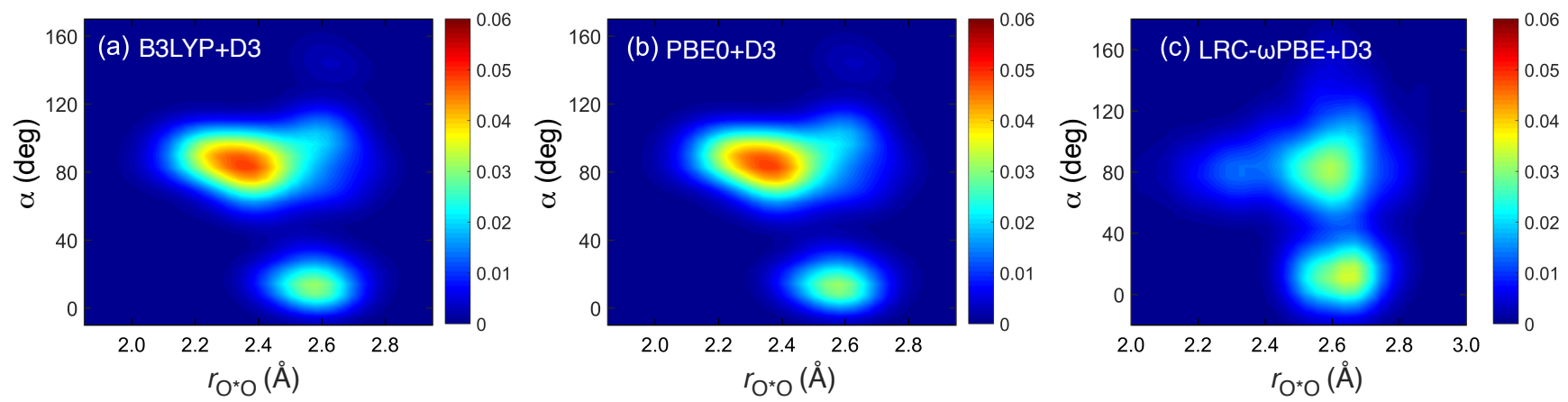

Figure 5: Two-dimensional joint distributions of $r_{\mathrm{O} * \mathrm{O}}$ and $\alpha$, for simulations using (a) B3LYP+D3, (b) PBE0+D3, and (c) LRC- $\omega$ PBE+D3. These geometrical parameters are defined in Fig. 1 .
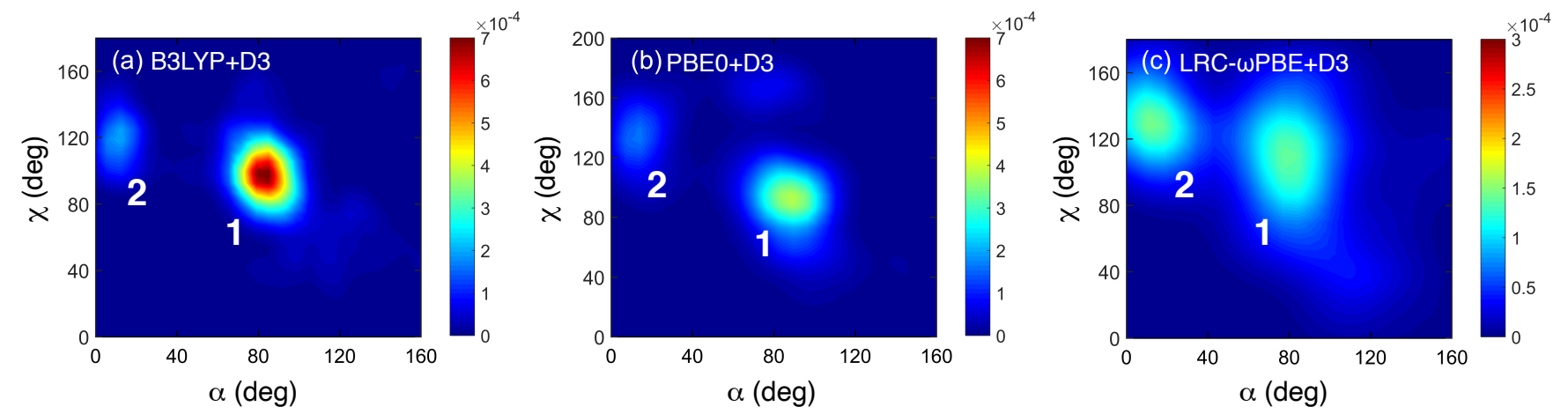

Figure 6: Two-dimensional joint distributions of $\alpha$ and $\chi$ (as defined in Fig. 1), for simulations using (a) B3LYP+D3, (b) PBE0+D3, and (c) LRC- $\omega$ PBE+D3. The two prominent features are labeled "1" and "2" for convenient reference in the text.

+D3. For the former, the most probable parameters are $r_{\mathrm{O}^{*} \mathrm{O}} \leq 2.5 \AA$ and $70^{\circ} \leq \alpha \leq 110^{\circ}$, which looks like the classical hemibond as suggested by Chipman! $\frac{29}{\text { (See }}$ Fig. 1]) The second feature in the probability distribution for these functionals appears between $r_{\mathrm{O}^{*} \mathrm{O}}=2.5 \AA$ and $r_{\mathrm{O}^{*} \mathrm{O}}=2.7 \AA$, with $\alpha<40^{\circ}$, and is characteristic of hydrogen bonding with ${ }^{\circ} \mathrm{OH}$ as the donor. The latter (hydrogen-bonded) feature remains roughly similar in simulations performed using LRC- $\omega \mathrm{PBE}+\mathrm{D} 3$, although the distribution of angles $\alpha$ is somewhat wider, consistent with a solvation environment that is less structured overall. The most significant difference relative to the B3LYP +D3 and PBE0+D3 simulations, however, occurs in the region $70^{\circ} \leq \alpha \leq 110^{\circ}$. Here, the probability distribution with $r_{\mathrm{O}^{*} \mathrm{O}}<2.5 \AA$ is diminished almost to zero, consistent with the lack of any feature in $g\left(r_{\mathrm{O}^{*} \mathrm{O}}\right)$ at smaller values of $r_{\mathrm{O}^{*} \mathrm{O}}$ than the hydrogen-bonding peak. Moreover, regarding the joint $\left(r_{\mathrm{O}^{*} \mathrm{O}}, \alpha\right)$ probability distribution in Fig. 5e, the boundary between the large- and small- $\alpha$ features in the distribution is not as sharp as it was in the case of B3LYP+D3 or PBE0+D3.

Joint distributions in the $(\alpha, \chi)$ are plotted in Fig. 6 . 
Here, the distributions obtained using B3LYP +D3 and PBE0 + D3 are weakly bimodal, with features that are labeled "1" and "2" in Fig. 6. The far more significant peak is feature 1 , appearing in the region where $70^{\circ} \leq \alpha \leq 110^{\circ}$ and $70^{\circ} \leq \chi \leq 120^{\circ}$, whereas feature 2 is much fainter and is characterized by $\alpha<30^{\circ}$ and $100^{\circ} \leq \chi \leq 130^{\circ}$. The latter is not a minor feature in the LRC- $\omega \mathrm{PBE}+\mathrm{D} 3$ simulations, however, but is comparable to feature 1. Based on Chipman's criteria for the hemibond (Fig. 1), it seems likely that feature 1 results predominantly from hemibonded configurations while feature 2 arises from hydrogen-bonded configurations. This is interesting in view of the fact that both features are prominent in the LRC- $\omega \mathrm{PBE}+\mathrm{D} 3$ simulation, whereas other considerations have pointed (perhaps) to less hemibonding in the solvation structure obtained using this functional. Comparison to the $\left(r_{\mathrm{O}^{*} \mathrm{O}}, \alpha\right)$ distributions in Fig. 5 suggests that because feature 2 is characterized by $\alpha \leq 30^{\circ}$, that it is also likely characterized by $r_{\mathrm{O}^{*} \mathrm{O}}>2.5 \AA$, making it difficult to ascribe hemibonding character to this feature in the $(\alpha, \chi)$ distributions.

Based on analysis of the B3LYP +D3 and PBE0+D3 data, suitable geometric criteria for the hemibond appear to be $r_{\mathrm{O}^{*} \mathrm{O}}<2.5 \AA, 70^{\circ} \leq \alpha \leq 110^{\circ}$, and $70^{\circ} \leq \chi \leq$ $120^{\circ}$, although the range for $\chi$ is a rougher estimate because some hydrogen-bonded water molecules do attach to $\cdot \mathrm{OH}$ with similar values of $\chi$. (One-dimensional probability distributions in $\chi$ do not exhibit any clear separation into hydrogen-bonded and hemibonded parts; see Fig. S12.) However, the geometrical analysis attempted in this section has exposed some ambiguities, especially concerning hemibonding in the LRC- $\omega \mathrm{PBE}+\mathrm{D} 3$ simulations. In an attempt to resolve these, and to formulate a cleaner definition of hemibonding, we next look for an electronic rather than a geometrical definition.

\subsection{Spin charge distribution}

VandeVondele and Sprik ${ }^{8}$ showed that the distribution of spin charges (obtained via Mulliken analysis applied to the spin density $\left.\rho_{\alpha}-\rho_{\beta}\right)$ is useful to investigate hemibonding. Specifically, the sum $s_{\mathrm{OH}}$ of the spin charges on the $\mathrm{O}^{*}$ and $\mathrm{H}^{*}$ provides a measure of "spin leakage" (delocalization of the unpaired electron) onto the neighboring water molecules, as indicated by values $s_{\mathrm{OH}}<1$. We expect this leakage to be more pronounced for hemibonded structures with their characteristic three-electron bond. At the same time, artificial spin leakage is also a signature of SIE. In an attempt to use $s_{\mathrm{OH}}$ to obtain a consistent electronic definition of the hemibond, we will analyze all snapshots from the different simulations using a common density-functional approach, namely OT-LRC- $\omega \mathrm{PBE}+$ D3, although we tune this functional separately for each simulation. (This will be discussed in detail in the context of the electronic spectroscopy, in Section 4.

Histograms of $s_{\mathrm{OH}}$ from different trajectories are plotted in Fig. 7. normalized to unit area so that they may be interpreted as probability distributions. Each distribution is bimodal (albeit only weakly so in the LRC$\omega \mathrm{PBE}+\mathrm{D} 3$ case), with one peak around $0.85 e$ and the other around 0.95e (or 0.87e and 0.97e in the case of LRC- $\omega \mathrm{PBE}+\mathrm{D} 3)$. We associate the first of these peaks, corresponding to greater spin leakage, with hemibonded configurations, which is confirmed by joint probability distributions in $r_{\mathrm{O}^{*} \mathrm{O}}$ and $s_{\mathrm{OH}}$ (Fig. S14), indicating that the distribution centered at the smaller value of $s_{\mathrm{OH}}$ is associated with $r_{\mathrm{O}^{*} \mathrm{O}}<2.5 \AA$, whereas the other feature, corresponding to the radical center being almost entirely localized on the $\cdot \mathrm{OH}$ moiety, is primarily associated with $r_{\mathrm{O}^{*} \mathrm{O}}=2.5-2.7 \AA$. We note that the fraction of the snapshots corresponding to the hemibonded feature decreases in the order

$$
\mathrm{B} 3 \mathrm{LYP}+\mathrm{D} 3>\mathrm{PBE} 0+\mathrm{D} 3>\mathrm{LRC}-\omega \mathrm{PBE}+\mathrm{D} 3,
$$

from $\approx 40 \%$ of the total for B3LYP $+\mathrm{D} 3$ to $\approx 10 \%$ for LRC- $\omega \mathrm{PBE}+\mathrm{D} 3$, suggesting the same trend for the relative population of the hemibonded species. Similar behavior is observed upon going from simulations with BLYP to those with BLYP-SIC ${ }^{8}$ For the LRC- $\omega$ PBE + D3 simulation, the joint probability distribution in $\left(r_{\mathrm{O}^{*} \mathrm{O}}, s_{\mathrm{OH}}\right)$ is peaked strongly around $r_{\mathrm{O}^{*} \mathrm{O}}=2.65 \AA$ and $s_{\mathrm{OH}}=0.97 e$ (see Fig. S14 ), with only a faint tail corresponding to smaller values of $s_{\mathrm{OH}}$ and shorter values of $r_{\mathrm{O} * \mathrm{O}}$.

The question arises whether these hemibonded configurations constitute inherent structures, ${ }^{91}$ or are instead fluctuations that are only accessed transiently, perhaps representing saddle points or unstable minima on the underlying potential energy surface. fluctuations. To address this, we took a few snapshots from the B3LYP+D3 and PBE0+D3 simulations and quenched them using a few hundred steps of QM/MM energy minimization with periodic boundary conditions. No significant change in the value of $s_{\mathrm{OH}}$ is observed over the course of the optimizations, as shown for several examples in Fig. S8. It therefore appears that the hemibond is indeed an inherent structure of $\cdot \mathrm{OH}(\mathrm{aq})$, rather than an energetic saddle point that is accessed only fleetingly.

\section{Electronic Spectroscopy}

\subsection{Ensemble-averaged results}

Based on EOM-CCSDT calculations performed on binary $\mathrm{HO} \cdots \mathrm{H}_{2} \mathrm{O}$ complexes, Chipman ${ }^{29[61}$ has suggested that the feature around $5.4 \mathrm{eV}(230 \mathrm{~nm})$ in the absorption spectrum of $\cdot \mathrm{OH}(\mathrm{aq})$ arises from a CT transition whose oscillator strength is $\approx 10 \times$ larger in hemibonded configurations than it is in hydrogen-bonded structures. This result was not affected in any meaningful way by the addition of a continuum solvation model ${ }^{61}$

Here, we compute the absorption spectrum of $\cdot \mathrm{OH}(\mathrm{aq})$ in full atomistic detail using TD-DFT. As described in 

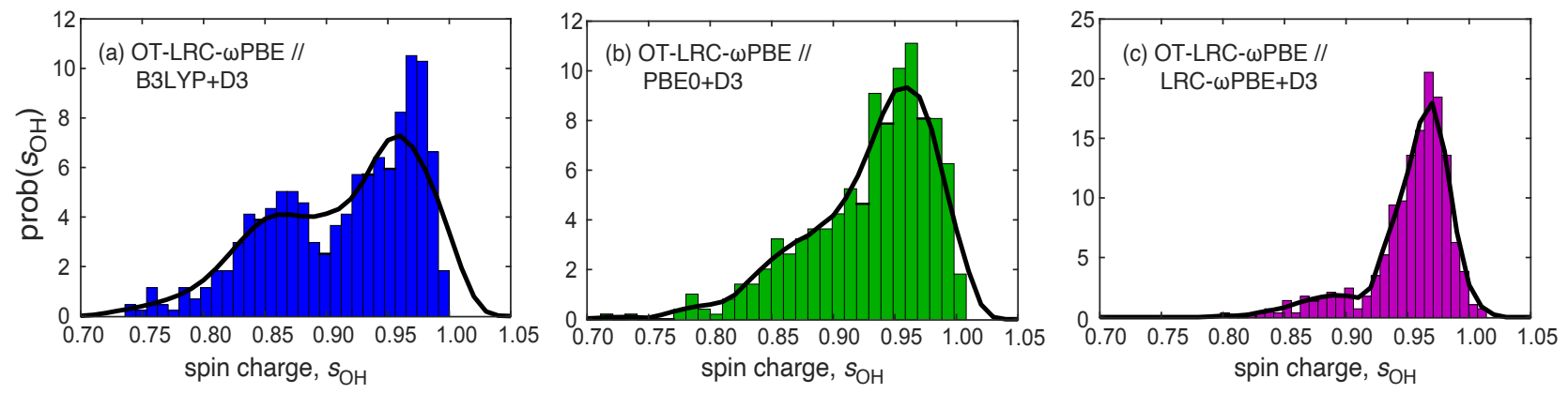

Figure 7: Histograms for the total spin charge $s_{\mathrm{OH}}$ on the $\cdot \mathrm{OH}$ moiety, for simulations performed using (a) B3LYP $+\mathrm{D} 3$, (b) PBE0+D3, and (c) LRC- $\omega$ PBE+D3. The spin charges are computed at the OT-LRC- $\omega$ PBE + D3/6-31+G* level, with $\omega$ tuned separately for each simulation. Black curves are fits to the distribution, which can be interpreted as probability distributions for $s_{\mathrm{OH}}$.

Table 3: TD-DFT excitation energies (in eV) corresponding to the absorption maxima for $\cdot \mathrm{OH}(\mathrm{aq})$ computed using various density functionals, for three different QM/MM trajectories. Footnotes indicate the range-separation parameters used in each case.

\begin{tabular}{lccc}
\hline \hline TD-DFT & \multicolumn{3}{c}{ Trajectory } \\
\cline { 2 - 4 } Functional & B3LYP & PBE0 & LRC- $\omega P B E^{a}$ \\
\hline \hline B3LYP & 3.285 & - & - \\
PBE0 & - & 3.496 & - \\
CAM-B3LYP & 4.434 & 4.400 & 4.351 \\
OT-LRC- $\mu$ BLYP & $4.540^{b}$ & $4.550^{c}$ & $4.502^{d}$ \\
OT-LRC- $\omega$ PBE & $4.644^{e}$ & $4.704^{f}$ & $4.877^{g}$ \\
BH\&HLYP & 5.110 & 5.395 & 6.003 \\
\hline \hline${ }_{\omega} \omega=0.380$ & $a_{0}^{-1} \cdot{ }^{b} \mu=0.387$ & $a_{0}^{-1} \cdot{ }^{c} \mu=0.386$ & $a_{0}^{-1} \cdot$ \\
${ }^{d} \mu=0.371$ & $a_{0}^{-1} \cdot{ }^{e} \omega=0.383$ & $a_{0}^{-1} \cdot{ }^{f}{ }_{\omega} \omega=0.382$ & $a_{0}^{-1}$. \\
$g_{\omega}=0.369$ & $a_{0}^{-1} \cdot$
\end{tabular}

Section 2.2 , these calculations are carried out within a $\mathrm{QM} / \mathrm{MM}$ formalism using four different functionals and the $6-31++\mathrm{G}^{*}$ basis set. Figure 8 shows a comparison of the spectra thus obtained. Absorption maxima are listed in Table 3, which also provides the optimally-tuned range separation parameters for each functional. Spectra for different trajectory simulations are computed using the same set of functionals, but the LRC- $\omega$ PBE and LRC$\mu$ BLYP functionals are tuned separately for each simulation so that condition in Eq. (4) is satisfied in an average way for a given set of structural snapshots from the same simulation.

Spectra from the OT-LRC functionals exhibit absorption maxima of 4.5-4.8 eV, which represents a significant red-shift with respect to the experimental maximum at $5.4 \mathrm{eV}[62] 65$ Results with CAM-B3LYP are consistently $\approx 0.15 \mathrm{eV}$ to the red of the OT-LRC results, although we do not place much significance on a shift of this magnitude, which is smaller than the intrinsic accuracy of TD-DFT even in favorable cases ${ }^{[2]}$ For the B3LYP+D3 and PBE0+D3 trajectories, we also performed TD-DFT calculations with the same functional that was used for the QM/MM simulation itself, and these results lie signif- icantly to the red of the CAM-B3LYP spectrum, consistent with the appearance of spurious, low-energy states involving $\mathrm{CT}$ either to or from the solvent 93

In contrast to these results, all of which are redshifted relative to experiment, the BH\&HLYP functional predicts absorption at higher energies. In fact, TD-BH\&HLYP spectra obtained from the PBE0 + D3 and B3LYP + D3 trajectories are a good match to the experimental absorption maximum. This is not altogether surprising given that BH\&HLYP was previously shown to match gas-phase EOM-CCSDT results for $\mathrm{HO} \cdot \cdots \mathrm{H}_{2} \mathrm{O}{ }^{29}$ When applied to the LRC- $\omega \mathrm{PBE}+\mathrm{D} 3$ trajectory, however, TD-BH\&HLYP calculations predict an absorption maximum $\approx 0.6 \mathrm{eV}$ higher than experiment. To the extent that the favorable comparison between TD-BH\&HLYP and EOM-CCSDT in binary complexes may be carried over to larger hydrated $\cdot \mathrm{OH}$ models (and TD-BH\&HLYP contains enough exact exact to avoid many of the spurious CT problems exhibited by functionals such as B3LYP), ${ }^{96}$ this overestimation of the absorption maximum for LRC- $\omega \mathrm{PBE}+\mathrm{D} 3$ configurations may point to a too-small role for hemibonded configurations in that particular simulation. This will become more evident when we decompose the absorption spectrum into contributions from hemibonded structures and non-hemibonded structures, in Section 4.2 .

Whereas the spectra in Fig. 8 are normalized, for direct comparison to the vertical excitation spectra reported elsewhere, we present the ensemble-averaged oscillator strength distribution in Fig. S18, without normalization. For B3LYP and PBE0 the oscillator strength at the absorption maximum lies in the range 0.09-0.10, comparable to published results for $\mathrm{HO}^{\cdots} \cdots \mathrm{H}_{2} \mathrm{O}, 61$ and $5-6 \times$ larger than the absolute oscillator strength computed for LRC- $\omega$ PBE at its absorption maximum. Given the enhanced oscillator strength that is associated with transitions from hemibonded geometries, ${ }^{29161}$ this further suggests that hemibonded configurations are a minority population in the LRC- $\omega \mathrm{PBE}+\mathrm{D} 3$ trajectory, consistent with other evidence documented above.

Examining the nature of the transitions that are re- 

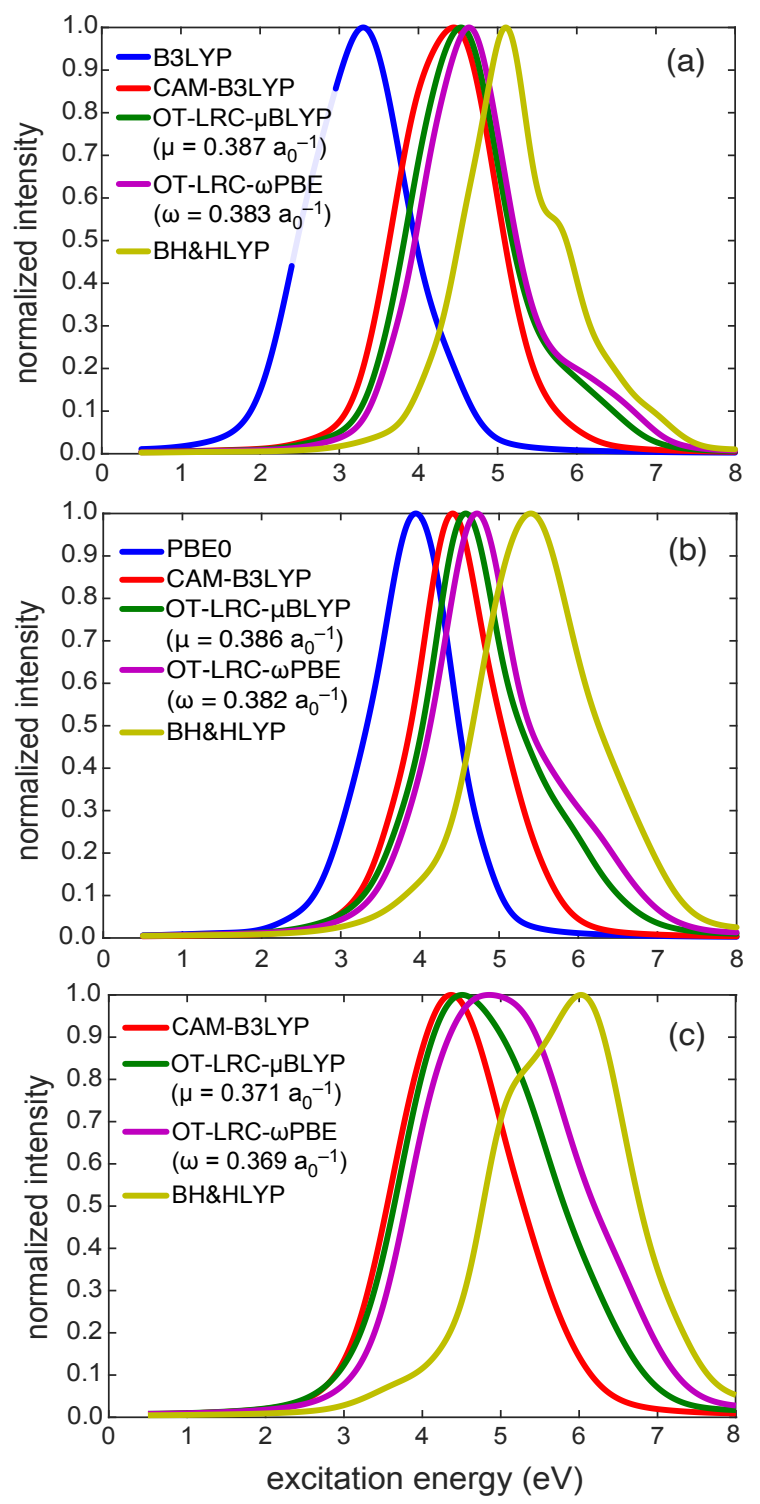

Figure 8: $\quad$ Ensemble-averaged absorption spectra for $\bullet \mathrm{OH}(\mathrm{aq})$ computed using TD-DFT with various functionals as indicated, for $\mathrm{QM} / \mathrm{MM}$ trajectory data obtained from (a) $\mathrm{B} 3 \mathrm{LYP}+\mathrm{D} 3$, (b) PBE0+D3, or (c) LRC- $\omega \mathrm{PBE}+\mathrm{D} 3$ simulations.

sponsible for the $\cdot \mathrm{OH}(\mathrm{aq})$ absorption spectrum, we find that the ones with largest oscillator strength are $\mathrm{CT}$ excitations, $1 b_{2}\left(\mathrm{H}_{2} \mathrm{O}\right) \rightarrow 2 p \pi(\cdot \mathrm{OH})$. The donor orbital is the frontier lone pair of water and the acceptor is the SOMO of the radical, in a hemibonded arrangement. Analogous CT excitations from hydrogen-bonded water molecules do appear but with much smaller oscillator strengths. The lower absolute oscillator strength in the ensembleaveraged spectrum obtained from LRC- $\omega \mathrm{PBE}+\mathrm{D} 3$ trajectories (Fig. S18) is therefore consistent with the appearance of fewer hemibonded configurations in that simulation, as compared to the B3LYP+D3 and PBE0+D3 simulations.

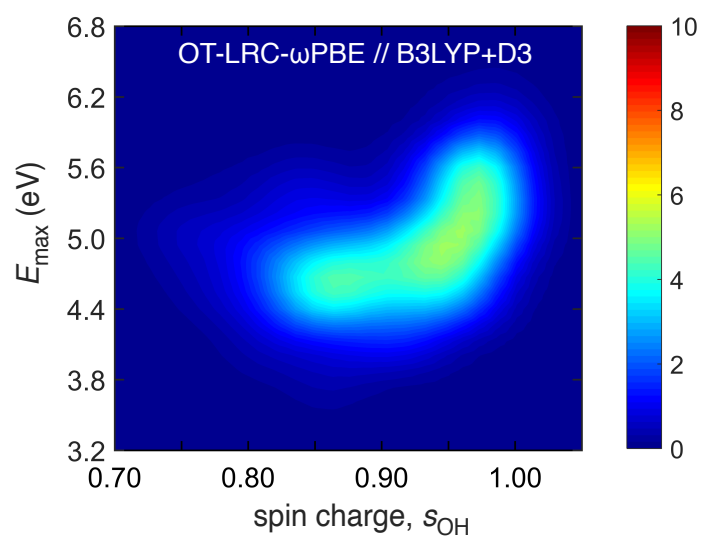

Figure 9: Joint distribution of the spin charge on the hydroxyl moiety $\left(s_{\mathrm{OH}}\right)$ versus the absorption maximum $E_{\max }$. Calculations were performed using the OT-LRC- $\omega$ PBE functional on geometries obtained from QM/MM simulations using B3LYP+D3.

\section{$4.2 \quad$ Spectral decompositions}

The bimodal nature of the probability distributions for coordinates $r_{\mathrm{O}^{*} \mathrm{O}}$ and $\alpha$, and for the ${ }^{\circ} \mathrm{OH}$ spin charge $s_{\mathrm{OH}}$, strongly suggests that the trajectories consist mainly of two types of structures, hemibonded and not hemibonded. This motivates us to attempt a decomposition of the simulated absorption spectra into contributions from each of these two populations, in order to test Chipman's idea that a minority population of hemibonded structures is primarily responsible for the absorption feature at $5.4 \mathrm{eV} \underline{\underline{29}}$

Bimodal distributions of $s_{\mathrm{OH}}$ can be separated by setting a cutoff value around $0.90-0.92 e$ (see Fig. 7), and $r_{\mathrm{O}^{*} \mathrm{O}}$ distributions can be separated with a cutoff of $r_{\mathrm{O}^{* \mathrm{O}}}=2.5 \AA$ (see Fig. 5). For an individual liquid configuration, we can estimate the absorption maximum $E_{\max }$ as a weighted average of vertical excitation energies,

$$
E_{\max }=\frac{\sum_{i=1}^{n} f_{0 i} E_{i}}{\sum_{j=1}^{n} f_{0 j}}
$$

which allows us to construct joint distributions $\left(s_{\mathrm{OH}}, E_{\max }\right)$ and $\left(r_{\mathrm{O}^{*} \mathrm{O}}, E_{\max }\right)$. The former is depicted for the B3LYP+D3 simulation in Fig. 9, using the OTLRC- $\omega$ PBE functional to compute $n=15$ excited states. (Other distributions can be found in Figs. S15 and S16.) Using this distribution, we may examine hypothetical scenarios in which the simulation is restricted to particular values of $s_{\mathrm{OH}}$, and from Fig. 9 it is clear the restriction $s_{\mathrm{OH}} \leq 0.92 e$ affords an absorption maximum $E_{\max } \approx 4.7 \mathrm{eV}$. This is perfectly aligned with the absorption maximum computed with the same functional when averaged over all configurations; see Fig. 8(a). The same is true for the PBE0+D3 simulation [see Fig. S15(b)], although this analysis is more ambiguous for the LRC$\omega \mathrm{PBE}+\mathrm{D} 3$ simulation [Fig. $\mathrm{S} 15(\mathrm{c})]$, because there are very few snapshots with $s_{\mathrm{OH}} \leq 0.92 e$. 

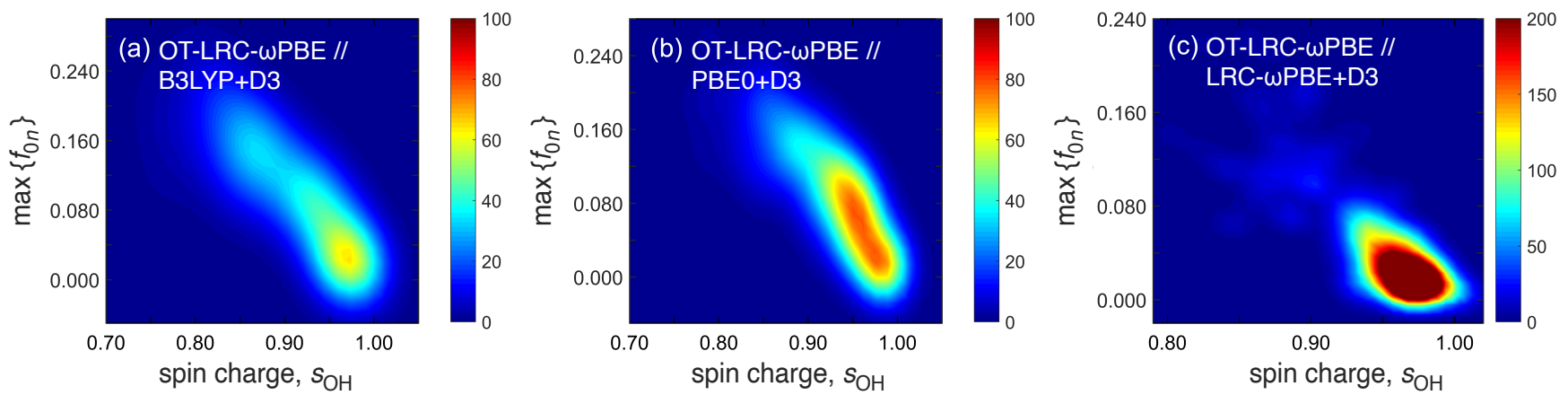

Figure 10: Joint distribution of the spin charge on the hydroxyl moiety $\left(s_{\mathrm{OH}}\right)$ versus the largest oscillator strength $\left(\max \left\{f_{0 n}\right\}\right)$, for QM/MM simulations performed using (a) B3LYP+D3, (b) PBE0+D3, and (c) LRC- $\omega$ PBE+D3.

That said, the largest oscillator strength obtained from individual snapshots exhibit correlations with the instantaneous value of $s_{\mathrm{OH}}$ that are obvious even for the LRC- $\omega \mathrm{PBE}+\mathrm{D} 3$ simulation. Joint distributions $\left(s_{\mathrm{OH}}, \max \left\{f_{0 n}\right\}\right)$ are plotted in Fig. 10, displaying these trends. Decreasing $s_{\mathrm{OH}}$, which signals spin delocalization onto the water molecules (or in other words, candidate structures for hemibonding) clearly correlates with a larger values for the maximum oscillator strength. This is true even for the LRC- $\omega \mathrm{PBE}+\mathrm{D} 3$ simulation where the spin leakage is smallest.

In view of this trend, we applied a cutoff $s_{\mathrm{OH}}=0.95 e$ to snapshots from each trajectory, in order to partition each spectrum into contributions from hemibonded and non-hemibonded configurations. These decompositions are shown in Fig. 11. For the B3LYP+D3 and PBE0+D3 trajectories, $63 \%$ and $57 \%$ (respectively) of the snapshots have $s_{\mathrm{OH}} \leq 0.95 e$, but these configurations are responsible for essentially the entire absorption spectrum. For the LRC- $\omega \mathrm{PBE}+\mathrm{D} 3$ simulation only $33 \%$ of the configurations have $s_{\mathrm{OH}} \leq 0.95 e$, nevertheless this minority population contributes roughly equally to the spectral intensity alongside the $67 \%$ of snapshots for which there is little or no spin leakage.

A similar analysis can be applied using $r_{\mathrm{O}^{*} \mathrm{O}}$ to partition the spectra, rather than $s_{\mathrm{OH}}$, as indeed a joint distribution $\left(r_{\mathrm{O}^{*} \mathrm{O}}, s_{\mathrm{OH}}\right)$ shows strong correlation between the two as shown in Fig. S14. Snapshots with $r_{\mathrm{O}^{*} \mathrm{O}} \leq 2.5 \AA$ are generally associated with $s_{\mathrm{OH}} \leq 0.95 e$, and restricting the averaging to only those snapshots with $r_{\mathrm{O}^{*} \mathrm{O}} \leq$ $2.5 \AA$ retains approximately the same absorption maximum as unrestricted averaging (Fig. S16). Spectral decompositions, according to whether $r_{\mathrm{O} * \mathrm{O}}$ is greater than or less than $2.5 \AA$ are shown in Fig. S19 and the results of this partition closely result what is shown in Fig. 11. Specifically, for the two global hybrid functionals, nearly the entirety of the oscillator strength is carried by configurations with $r_{\mathrm{O}^{*} \mathrm{O}} \leq 2.5 \AA$, whereas for the LRC- $\omega \mathrm{PBE}$ +D3 simulation this is a minority population but is still responsible for approximately half of the integrated oscillator strength.

\section{Conclusions}

We have reported on $\mathrm{QM} / \mathrm{MM}$ simulations of $\cdot \mathrm{OH}(\mathrm{aq})$ using various hybrid and range-separated hybrid functionals. Hemibonded structures of the radical appear as one of the main solvation motifs for simulations using B3LYP+D3 or PBE0+D3, as indicated by oxygen-oxygen RDFs and by analysis of the spin density, which is more delocalized in hemibonded configurations. This is a different conclusion than was reached in recent ai MD simulations of $\cdot \mathrm{OH}(\mathrm{aq})$ using the same functionals, ${ }^{21}$ where no hemibonded configurations are reported at all. The theoretical framework is somewhat different in the present work, nevertheless this disparity remains a subject for further investigation.

For QM/MM simulations using LRC- $\omega \mathrm{PBE}+\mathrm{D} 3$, hemibonded configurations are significantly suppressed but not absent. Shorter trajectories at the BLYP + D3, BH\&HLYP + D3, and HF + D3 levels of theory highlight the profound impact of exact exchange on the structure of the solvated radical. Simulations using the GGA functional BLYP + D3 exhibit a pronounced shoulder in $g\left(r_{\mathrm{O}^{*} \mathrm{O}}\right)$ at small values of $r_{\mathrm{O}^{*} \mathrm{O}}$, a feature that has long been taken as the signature of a hemibond 8 This feature is present, albeit less pronounced, in the B3LYP+ D3 and PBE0 + D3 trajectories but is absent for other functionals considered here, each of which has a larger fraction of exact exchange. Even for trajectories where this feature is absent, however, we nevertheless observe a strong correlation between oscillator strengths computed for the low-energy excited states and the spin charge $s_{\mathrm{OH}}$ on the $\cdot \mathrm{OH}$ moiety. This suggests that in all of the simulations, "spin leakage" (delocalization) onto the water molecules is associated with enhanced absorption at ultraviolet wavelengths.

Ensemble-averaged absorption spectra of $\cdot \mathrm{OH}(\mathrm{aq})$ were computed from the aforementioned trajectories using a variety of TD-DFT approaches. Notably, TDBH\&HLYP calculations have previously been shown to afford good agreement with benchmark transition energies for the binary $\mathrm{HO} \cdot \cdots \mathrm{H}_{2} \mathrm{O}$ complex ${ }^{29}$ In the present work, this functional affords the best agreement with the 

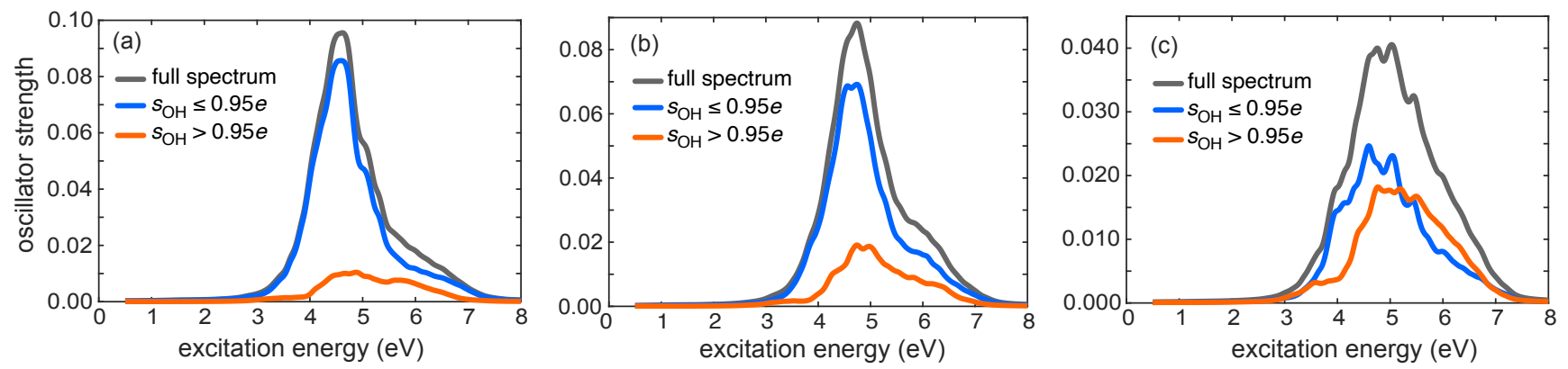

Figure 11: TD-DFT absorption spectra computed using the OT-LRC- $\omega$ PBE functional and separated into two contributions according to the value of the spin charge on the hydroxyl moiety, $s_{\mathrm{OH}}$. Results are shown for (a) the B3LYP+D3 trajectory, (b) the PBE0+D3 trajectory, and (c) the LRC- $\omega$ PBE+D3 trajectory.

experimental absorption maximum when the underlying solvent configurations are taken from simulations using the B3LYP+D3 and PBE0+D3 functionals. In contrast, TD-BH\&HLYP calculations overestimate the experimental absorption maximum by $\approx 0.6 \mathrm{eV}$ when applied to the LRC- $\omega$ PBE + D3 trajectory, suggesting that the paucity of hemibonded structures in the latter leads to an artificial blue-shift in the spectrum.

Chipman ${ }^{29}$ has suggested that the absorption band of $\cdot \mathrm{OH}(\mathrm{aq})$ at $230 \mathrm{~nm}(5.4 \mathrm{eV})$, which is strongly blueshifted from the absorption maximum of gas-phase $\cdot \mathrm{OH}$ (at $307 \mathrm{~nm}$ or $4.0 \mathrm{eV}$ ), is a result of solvent-to-radical CT bands whose oscillator strengths are quite large compared to those for excitations localized on the radical, leaving the radical-localized transitions of $\cdot \mathrm{OH}(\mathrm{aq})$ closer to the gas-phase absorption energy. In the present work, we evaluated this hypothesis by partitioning the ensembleaveraged spectra in several ways, according to thresholds applied to either the spin charge $s_{\mathrm{OH}}$, which affords an electronic definition of the hemibond, or else to the distance $r_{\mathrm{O}^{*} \mathrm{O}}$, which provides a geometric definition. By either definition, nearly the entire oscillator strength of the spectrum simulated using either B3LYP+D3 or PBE0 $+\mathrm{D} 3$ trajectories is carried by hemibonded configurations of the radical. For the LRC- $\omega \mathrm{PBE}+\mathrm{D} 3$ simulations, such configurations represent a minority species but are still responsible for approximately half of the integrated oscillator strength.
The hypothesis put forward by Chipman therefore appears to be on very solid footing, despite lingering questions regarding the prevalence of the hydroxyl-water hemibond. Further work is necessary to resolve these ambiguities, and to ascertain whether the hemibond is a self-interaction artifact (as has long been the conventional wisdom),$\frac{8}{\text { or }}$ whether finite-size effects may affect this conclusion (as has recently been demonstrated) ${ }^{12} \mathrm{~A}$ third possibility is that a delicate interplay between these two effects can alter the structure of $\cdot \mathrm{OH}(\mathrm{aq})$; indeed, the present simulations suggest that the energetics of the hemibond are sensitive to both factors, and that the true physical structure of the aqueous radical may lie near an inflection point in the space of simulation parameters. In that case, relatively minor modifications to the computational protocol might manifest as dramatically different predictions for the solvation structure. This is not without precedent for an aqueous radical. $28 \mid 99[101$ Additional simulations are planned to address this issue.

\section{Acknowledgements}

This work was supported by National Science Foundation grant nos. CHE-1665322 and CHE-1955282. Calculations were performed at the Ohio Supercomputer Center under project PAA-0003.
1 B. C. Garrett, D. A. Dixon, D. M. Camaioni, D. M. Chipman, M. A. Johnson, C. D. Jonah, G. A. Kimmel, J. H. Miller, T. N. Rescigno, P. J. Rossky, S. S. Xantheas, S. D. Colson, A. H. Laufer, D. Ray, P. F. Barbara, D. M. Bartels, K. H. Becker, K. H. Bowen, Jr., S. E. Bradforth, I. Carmichael, J. V. Coe, L. R. Corrales, J. P. Cowin, M. Dupuis, K. B. Eisenthal, J. A. Franz, M. S. Gutowski, K. D. Jordan, B. D. Kay, J. A. LaVerne, S. V. Lymar, T. E. Madey, C. W. McCurdy, D. Meisel, S. Mukamel, A. R. Nilsson, T. M. Orlando, N. G. Petrik, S. M. Pimblott, J. R. Rustad, G. K. Schenter, S. J. Singer, A. Tok- makoff, L.-S. Wang, C. Wittig, and T. S. Zwier, Role of water in electron-initiated processes and radical chemistry: Issues and scientific advances, Chem. Rev., 2005, 105, 355-389.

2 H. Herrmann, D. Hoffmann, T. Schaefer, P. Bräuer, and A. Tilgner, Tropospheric aqueous-phase free-radical chemistry: Radical sources, spectra, reaction kinetics and prediction tools, Comput. Phys. Commun., 2010, 11, 3796-3822.

3 I. S. A. Isaksen and S. B. Dalsøren, Getting a better estimate of an atmospheric radical, Science, 2011, 331, 38- 
39.

4 B. Lipinski, Hydroxyl radical and its scavengers in health and disease, Oxid. Med. Cell. Longev., 2011, 2011, 1-9.

${ }^{5}$ S. Gligorovski, R. Strekowski, S. Barbati, and D. Vione, Environmental implications of hydroxyl radicals $\left({ }^{\bullet} \mathrm{OH}\right)$, Chem. Rev., 2015, 115, 13051-13092, Erratum: ibid. 118, 2296 (2018).

${ }^{6}$ P. Vassilev, M. J. Louwerse, and E. J. Baerends, Ab initio molecular dynamics simulation of the $\mathrm{OH}^{\bullet}$ radical in liquid water, Chem. Phys. Lett., 2004, 398, 212-216.

7 P. Vassilev, M. J. Louwerse, and E. J. Baerends, Hydroxyl radical and hydroxide ion in liquid water: A comparative electron density functional study, J. Phys. Chem. B, 2005, 109, 23605-23610.

8 J. VandeVondele and M. Sprik, A molecular dynamics study of the hydroxyl radical in solution applying selfinteraction-corrected density functional methods, Phys. Chem. Chem. Phys., 2005, 7, 1363-1367.

9 J. M. Khalack and A. P. Lyubartsev, Solvation structure of hydroxyl radical by Car-Parrinello molecular dynamics, J. Phys. Chem. A, 2005, 109, 378-386.

10 I. M. Svishchev and A. Y. Plugatyr, Hydroxyl radical in aqueous solution: Computer simulation, J. Phys. Chem. B, 2005, 109, 4123-4128.

11 R. D'Auria, I.-F. W. Kuo, and D. J. Tobias, Ab initio molecular dynamics simulation of the $\mathrm{OH}^{-}$radical in liquid water, J. Phys. Chem. A, 2008, 112, 4644-4650.

12 E. Codorniu-Hernández and P. G. Kusalik, Insights into the solvation and mobility of the hydroxyl radical in aqueous solution, J. Chem. Theory Comput., 2011, 7, 37253732 .

13 E. Codorniu-Hernández and P. G. Kusalik, Mobility mechanism of hydroxyl radicals in aqueous solution via hydrogen transfer, J. Am. Chem. Soc., 2012, 134, 532538.

14 E. Codorniu-Hernández and P. G. Kusalik, Hydroxyl radicals in ice: Insights into local structure and dynamics, Phys. Chem. Chem. Phys., 2012, 14, 11639-11650.

15 E. Codorniu-Hernández, A. D. Boese, and P. G. Kusalik, The hemibond as an alternative condensed phase structure for the hydroxyl radical, Can. J. Chem, 2013, 91, 544-551.

16 A. Pabis, J. Szala-Bilnik, and D. Swiatla-Wojcik, Molecular dynamics study of the hydration of the hydroxyl radical at body temperature, Phys. Chem. Chem. Phys., 2011, 13, 9458-9468.

17 J. Szala-Bilnik and D. Swiatla-Wojcik, Hydration of $\mathrm{OH}$ radical in high temperature water, J. Mol. Liq., 2011, 164, 34-38.

18 D. Swiatla-Wojcik and J. Szala-Bilnik, Mechanism of $\mathrm{OH}$ radical hydration: A comparative computational study of liquid and supercritical solvent, J. Chem. Phys., 2012, 136, 064510:1-10.

19 A. Genova, D. Ceresoli, and M. Pavanello, Avoiding fractional electrons in subsystem DFT based ab-initio molecular dynamics yields accurate models for liquid water and solvated $\mathrm{OH}$ radical, J. Chem. Phys., 2016, 144, 234105:1-8.

20 G. F. von Rudorff, R. Jakobsen, K. M. Rosso, and J. Blumberger, Improving the performance of hybrid functional-based molecular dynamics simulation through screening of Hartree-Fock exchange forces, J. Chem. Theory Comput., 2017, 13, 2178-2184.

21 C. Apostolidou, $\mathrm{OH}$ radical in water from ab initio molec- ular dynamics simulation employing hybrid functionals, J. Chem. Phys., 2019, 151, 064111:1-18.

${ }^{22}$ L. Kjellsson, K. D. Nanda, J.-E. Rubensson, G. Doumy, S. H. Southworthh, P. J. Ho, A. M. March, A. Al Haddad, Y. Kumagai, M.-F. Tu, R. D. Schaller, T. Debnath, M. S. Bin Mohd Yusof, C. Arnold, W. F. Schlotter, S. Moeller, G. Coslovich, J. D. Koralek, M. P. Minitti, M. L. Vidal, M. Simon, R. Santra, Z.-H. Loh, S. Coriani, A. I. Krylov, and L. Young, Resonant inelastic x-ray scatternig reveals hidden local transitions of the aqueous $\mathrm{OH}$ radical, Phys. Rev. Lett., 2020, 124, 236001:1-7.

${ }^{23}$ G. V. Buxton, C. L. Greenstock, W. P. Helman, and A. B. Ross, Critical review of rate constants for reactions of hydrated electrons, hydrogen atoms and hydroxyl radicals $\left({ }^{\bullet} \mathrm{OH} /{ }^{\bullet} \mathrm{O}\right)$ in aqueous solution, J. Phys. Chem. Ref. Data, 1988, 17, 513-886.

24 A. J. Elliot, D. R. McCracken, G. V. Buxton, and N. D. Wood, Estimation of rate constants for neardiffusion-controlled reactions in water at high temperatures, J. Chem. Soc. Faraday Trans., 1990, 86, 1539-1547.

25 N. Agmon, The Grotthuss mechanism, Chem. Phys. Lett., 1995, 244, 456-462.

26 N. Agmon, Mechanism of hydroxide mobility, Chem. Phys. Lett., 2000, 319, 247-252.

27 D. Marx, A. Chandra, and M. E. Tuckerman, Aqueous basic solutions: Hydroxide solvation, structural diffusion, and comparison to the hydrated proton, Chem. Rev., 2010, 110, 2174-2216.

28 J. M. Herbert, Structure of the aqueous electron, Phys. Chem. Chem. Phys., 2019, 21, 20538-20565.

29 D. M. Chipman, Hemibonding between hydroxyl radical and water, J. Phys. Chem. A, 2011, 115, 1161-1171.

30 M. Holz, S. R. Heil, and A. Sacco, Temperaturedependent self-diffusion coefficients of water and six selected molecular liquids for calibration in accurate ${ }^{1} \mathrm{H}$ NMR PFG measurements, Phys. Chem. Chem. Phys., 2000, 2, 4740-4742.

31 B. Braïda, P. C. Hiberty, and A. Savin, A systematic failing of current density functionals: Overestimation of twocenter three-electron bonding energies, J. Phys. Chem. A, 1998, 102, 7872-7877.

32 J. Gräfenstein, E. Kraka, and D. Cremer, Effect of the self-interaction error for three-electron bonds: On the development of new exchange-correlation functionals, Phys. Chem. Chem. Phys., 2004, 6, 1096-1112.

33 A. J. Cohen, P. Mori-Sanchez, and W. Yang, Challenges for density functional theory, Chem. Rev., 2012, 112, 289320 .

${ }^{34}$ P. Zawadzki, J. Rossmeisl, and K. W. Jacobsen, Electronic hole transfer in rutile and anatase $\mathrm{TiO}_{2}$ : Effect of a delocalization error in the density functional theory on the charge transfer barrier height, Phys. Rev. B, 2011, 84, 121203(R):1-4.

35 G. Pacchioni, F. Frigoli, D. Ricci, and J. A. Weil, Theoretical description of hole localization in a quartz $\mathrm{Al}$ center: The importance of exact electron exchange, Phys. Rev. B, 2000, 63, 054102:1-8.

36 J. Lægsgaard and K. Stokbro, Hole trapping at Al impurities in silica: A challenge for density functional theories, Phys. Rev. Lett., 2001, 86, 2834-2837.

37 M. d'Avezac, M. Calandra, and F. Mauri, Density functional theory description of hole-trapping in $\mathrm{SiO}_{2}$ : A selfinteraction-corrected approach, Phys. Rev. B, 2005, 71, 205210:1-5. 
38 M. Sodupe, J. Bertran, L. Rodríguez-Santiago, and E. J. Baerends, Ground state of the $\left(\mathrm{H}_{2} \mathrm{O}\right)_{2}^{+}$radical cation: DFT versus post-Hartree-Fock methods, J. Phys. Chem. A, 1999, 103, 166-170.

39 E. Livshits, R. S. Granot, and R. Baer, A density functional theory for studying ionization processes in water clusters, J. Phys. Chem. A, 2011, 115, 5735-5744.

40 Y. A. Mantz, F. L. Gervasio, T. Laino, and M. Parrinello, Charge localization in stacked radical cation DNA base pairs and the benzene dimer studied by self-interaction corrected density-functional theory, J. Phys. Chem. A, 2007, 111, 105-112.

41 J. M. Herbert and M. Head-Gordon, Calculation of electron detachment energies for water cluster anions: An appraisal of electronic structure methods, with application to $\left(\mathrm{H}_{2} \mathrm{O}\right)_{20}^{-}$and $\left(\mathrm{H}_{2} \mathrm{O}\right)_{24}^{-}$, J. Phys. Chem. A, 2005, 109, 5217-5229.

42 J. M. Herbert, The quantum chemistry of loosely bound electrons, in Reviews in Computational Chemistry, ed. A. L. Parill and K. Lipkowitz, Vol. 28; Wiley-VCH, 2015; chapter 8, pp. 391-517.

43 T. Frigato, J. VandeVondele, B. Schmidt, C. Schütte, and $\mathrm{P}$. Jungwirth, Ab initio molecular dynamics simulation of a medium-sized water cluster anion: From an interior to a surface-located excess electron via a delocalized state, J. Phys. Chem. A, 2008, 112, 6125-6133.

44 P. R. Tentscher and J. S. Arey, Binding in radical-solvent binary complexes: Benchmark energies and performance of approximate methods, J. Chem. Theory Comput., 2013, 9, 1568-1579.

45 E. R. Johnson, M. Salamone, M. Bietti, and G. A. DiLabio, Modeling noncovalent radical-molecule interactions using conventional density-functional theory: Beware erroneous charge transfer, J. Phys. Chem. A, 2013, 117, 947-952.

46 M.-C. Kim, E. Sim, and K. Burke, Ions in solution: Density corrected density functional theory (DC-DFT), J. Chem. Phys., 2014, 140, 18A528:1-10.

47 M.-C. Kim, H. Park, S. Son, E. Sim, and K. Burke, Improved DFT potential energy surfaces via improved densities, J. Phys. Chem. Lett., 2015, 6, 3802-3807.

48 A. D. Becke, Density-functional exchange-energy approximation with correct asymptotic behavior, Phys. Rev. A, 1988, 38, 3098-3100.

49 C. Lee, W. Yang, and R. G. Parr, Development of the Colle-Salvetti correlation-energy formula into a functional of the electron density, Phys. Rev. B, 1988, 37, 785-789.

50 A. D. Boese, N. L. Doltsinis, N. C. Handy, and M. Sprik, New generalized gradient approximation functionals, J. Chem. Phys., 2000, 112, 1670-1678.

51 C. Adamo and V. Barone, Toward reliable density functional methods without adjustable parameters: The PBE0 model, J. Chem. Phys., 1999, 110, 6158-6170.

52 O. A. Vydrov and G. E. Scuseria, Effect of the PerdewZunger self-interaction correction on the thermochemical performance of approximate density functionals, J. Chem. Phys., 2004, 121, 8187-8193.

53 D. R. Lonsdale and L. Goerigk, The one-electron selfinteraction error in 74 density functional approximations: A case study on hydrogenic mono- and dinuclear systems, Phys. Chem. Chem. Phys., 2020, 22, 15805-15830.

54 D. Rappoport, N. R. M. Crawford, F. Furche, and K. Burke, Approximate density functionals: Which should I choose?, in Encyclopedia of Inorganic Chemistry, ed. R. B. King, R. H. Crabtree, C. M. Lukehart, D. A. Atwood, and R. A. Scott; Wiley, 2006.

55 J. L. Bao, L. Gagliardi, and D. G. Truhlar, Self-interaction error in density functional theory: An appraisal, J. Phys. Chem. Lett., 2018, 9, 2353-2358.

56 A. D. Becke, Density-functional thermochemistry. III. The role of exact exchange, J. Chem. Phys., 1993, 98, $5648-5652$.

57 J. Heyd, G. E. Scuseria, and M. Ernzerhof, Hybrid functionals based on a screened Coulomb potential, J. Chem. Phys., 2003, 118, 8207-8215, Erratum: ibid. 124, 219906 (2006).

58 S. Grimme, J. Antony, S. Ehrlich, and H. Krieg, A consistent and accurate $a b$ initio parameterization of density functional dispersion correction (DFT-D) for the 94 elements $\mathrm{H}-\mathrm{Pu}, J$. Chem. Phys., 2010, 132, 154104:1-19.

59 O. Oldenberg and F. F. Rieke, Kinetics of OH radicals as determined by their absorption spectrum III. A quantitative test for free $\mathrm{OH}$; probabilities of transition, J. Chem. Phys., 1938, 6, 439-447.

60 S. Pellerin, J. M. Cormier, F. Richard, K. Musiol, and J. Chapelle, A spectroscopic diagnostic method using uv oh band spectrum, J. Phys. D: Appl. Phys., 1996, 29, 726.

61 D. M. Chipman, Absorption spectrum of $\mathrm{OH}$ radical in water, J. Phys. Chem. A, 2008, 112, 13372-13381.

62 J. K. Thomas, J. Rabani, M. S. Matheson, E. J. Hart, and S. Gordon, Absorption spectrum of the hydroxyl radical, J. Phys. Chem., 1966, 70, 2409-2410.

63 P. Pagsberg, H. Christensen, J. Rabani, G. Nilsson, J. Fenger, and S. O. Nielsen, Far-ultraviolet spectra of hydrogen and hydroxyl radicals from pulse radiolysis of aqueous solutions. direct measurement of the rate of $\mathrm{H}+$ H, J. Phys. Chem., 1969, 73, 1029-1038.

64 A. Treinin and E. Hayon, Charge transfer spectra of halogen atoms in water. correlation of the electronic transition energies of iodine, bromine, chlorine, hydroxyl, and hydrogen radicals with their electron affinities, J. Am. Chem. Soc., 1975, 97, 1716-1721.

65 G. Czapski and B. H. J. Bielski, Absorption spectra of the ${ }^{\bullet} \mathrm{OH}$ and $\mathrm{O}^{\bullet-}$ radicals in aqueous solutions, Radiat. Phys. Chem., 1993, 41, 503-505.

66 S. O. Nielsen, B. D. Michael, and E. J. Hart, Ultraviolet absorption spectra of $e_{\mathrm{aq}}^{-}, \mathrm{H}, \mathrm{OH}, \mathrm{D}$, and OD from pulse radiolysis of aqueous solutions, J. Phys. Chem., 1976, 80, 2482-2488.

67 I. Janik, D. M. Bartels, and C. D. Jonah, Hydroxyl radical self-recombination reaction and absorption spectrum in water up to $350^{\circ} \mathrm{C}, J$. Phys. Chem. A, 2007, 111, 18351843.

68 M.-K. Tsai, K. Kowalski, M. Valiev, and M. Dupuis, Signature $\mathrm{OH}$ absorption spectrum from cluster models of solvation: A solvent-to-solute charge transfer state, J. Phys. Chem. A, 2007, 111, 10478-10482.

69 M. Yamaguchi, Hemibonding of hydroxyl radical and halide anion in aqueous solution, J. Phys. Chem. A, 2011, 115, 14620-14628.

70 G. J. Hoffman, P. K. Gurunathan, J. S. Francisco, and L. V. Slipchenko, Excited states of $\mathrm{OH}-\left(\mathrm{H}_{2} \mathrm{O}\right)_{n}$ clusters for $n=1-4$ : An ab initio study, J. Chem. Phys., 2014, 141, 104315:1-9.

71 M. A. Rohrdanz, K. M. Martins, and J. M. Herbert, A long-range-corrected density functional that performs well for both ground-state properties and time-dependent density functional theory excitation energies, including 
charge-transfer excited states, J. Chem. Phys., 2009, 130, 054112:1-8.

72 Y. Shao, Z. Gan, E. Epifanovsky, A. T. B. Gilbert, M. Wormit, J. Kussmann, A. W. Lange, A. Behn, J. Deng, X. Feng, D. Ghosh, M. Goldey, P. R. Horn, L. D. Jacobson, I. Kaliman, R. Z. Khaliullin, T. Kús, A. Landau, J. Liu, E. I. Proynov, Y. M. Rhee, R. M. Richard, M. A. Rohrdanz, R. P. Steele, E. J. Sundstrom, H. L. Woodcock III, P. M. Zimmerman, D. Zuev, B. Albrecht, E. Alguire, B. Austin, G. J. O. Beran, Y. A. Bernard, E. Berquist, K. Brandhorst, K. B. Bravaya, S. T. Brown, D. Casanova, C.-M. Chang, Y. Chen, S. H. Chien, K. D. Closser, D. L. Crittenden, M. Diedenhofen, R. A. DiStasio Jr., H. Do, A. D. Dutoi, R. G. Edgar, S. Fatehi, L. Fusti-Molnar, A. Ghysels, A. Golubeva-Zadorozhnaya, J. Gomes, M. W. D. Hanson-Heine, P. H. P. Harbach, A. W. Hauser, E. G. Hohenstein, Z. C. Holden, T.-C. Jagau, H. Ji, B. Kaduk, K. Khistyaev, J. Kim, J. Kim, R. A. King, P. Klunzinger, D. Kosenkov, T. Kowalczyk, C. M. Krauter, K. U. Lao, A. Laurent, K. V. Lawler, S. V. Levchenko, C. Y. Lin, F. Liu, E. Livshits, R. C. Lochan, A. Luenser, P. Manohar, S. F. Manzer, S.-P. Mao, N. Mardirossian, A. V. Marenich, S. A. Maurer, N. J. Mayhall, C. M. Oana, R. Olivares-Amaya, D. P. O'Neill, J. A. Parkhill, T. M. Perrine, R. Peverati, P. A. Pieniazek, A. Prociuk, D. R. Rehn, E. Rosta, N. J. Russ, N. Sergueev, S. M. Sharada, S. Sharma, D. W. Small, A. Sodt, T. Stein, D. Stück, Y.-C. Su, A. J. W. Thom, T. Tsuchimochi, L. Vogt, O. Vydrov, T. Wang, M. A. Watson, J. Wenzel, A. White, C. F. Williams, V. Vanovschi, S. Yeganeh, S. R. Yost, Z.-Q. You, I. Y. Zhang, X. Zhang, Y. Zhao, B. R. Brooks, G. K. L. Chan, D. M. Chipman, C. J. Cramer, W. A. Goddard III, M. S. Gordon, W. J. Hehre, A. Klamt, H. F. Schaefer III, M. W. Schmidt, C. D. Sherrill, D. G. Truhlar, A. Warshel, X. Xu, A. AspuruGuzik, R. Baer, A. T. Bell, N. A. Besley, J.-D. Chai, A. Dreuw, B. D. Dunietz, T. R. Furlani, S. R. Gwaltney, C.-P. Hsu, Y. Jung, J. Kong, D. S. Lambrecht, W. Liang, C. Ochsenfeld, V. A. Rassolov, L. V. Slipchenko, J. E. Subotnik, T. Van Voorhis, J. M. Herbert, A. I. Krylov, P. M. W. Gill, and M. Head-Gordon, Advances in molecular quantum chemistry contained in the Q-Chem 4 program package, Mol. Phys., 2015, 113, 184-215.

73 R. Baer, E. Livshits, and U. Salzner, Tuned rangeseparated hybrids in density functional theory, Annu. Rev. Phys. Chem., 2010, 61, 85-109.

${ }^{74}$ W. L. Jorgensen, J. Chandrasekhar, J. D. Madura, R. W. Imprey, and M. L. Klein, Comparison of simple potential functions for simulating liquid water, J. Chem. Phys., 1983, 79, 926-935.

75 P. H. Hünenberger, Thermostat algorithms for molecular dynamics simulations, Adv. Polym. Sci, 2005, 173, 105149.

76 J. M. Herbert and M. Head-Gordon, Accelerated, energyconserving Born-Oppenheimer molecular dynamics via Fock matrix extrapolation, Phys. Chem. Chem. Phys., 2005, 7, 3269-3275.

77 Z. C. Holden, R. M. Richard, and J. M. Herbert, Periodic boundary conditions for QM/MM calculations: Ewald summation for extended Gaussian basis sets, J. Chem. Phys., 2013, 139, 244108:1-13, Erratum: ibid. 142, 059901:1-2 (2015).

78 Z. C. Holden, B. Rana, and J. M. Herbert, Analytic energy gradients for the QM/MM-Ewald method using atomic charges derived from the electrostatic potential: Theory, implementation, and application to ab initio molecular dynamics of the aqueous electron, J. Chem. Phys., 2019, 150, 144115:1-20.

79 S. Dasgupta, B. Rana, and J. M. Herbert, Ab initio investigation of the resonance Raman spectrum of the hydrated electron, J. Phys. Chem. B, 2019, 123, 8074-8084.

80 C. M. Breneman and K. B. Wiberg, Determining atomcentered monopoles from molecular electrostatic potentials. The need for high sampling density in formamide conformational analysis, J. Comput. Chem., 1990, 11, 361-373.

81 M. M. Francl and L. E. Chirlian, The pluses and minuses of mapping atomic charges to electrostatic potentials, in Reviews in Computational Chemistry, ed. K. B. Lipkowitz and D. B. Boyd, Vol. 14; Wiley-VCH, New York, 2000; chapter 1, pp. 1-32.

82 J. M. Herbert, L. D. Jacobson, K. U. Lao, and M. A. Rohrdanz, Rapid computation of intermolecular interactions in molecular and ionic clusters: Self-consistent polarization plus symmetry-adapted perturbation theory, Phys. Chem. Chem. Phys., 2012, 14, 7679-7699.

83 M. Zheng and M. P. Waller, Adaptive quantum mechanics/molecular mechanics methods, WIREs Comput. Mol. Sci., 2016, 6, 369-385.

84 C. N. Rowley and B. Roux, The solvation structure of $\mathrm{Na}^{+}$and $\mathrm{K}^{+}$in liquid water determined from high level $a b$ initio molecular dynamics simulations, J. Chem. Theory Comput., 2012, 8, 3526-3535.

85 S. Hirata and M. Head-Gordon, Time-dependent density functional theory within the Tamm-Dancoff approximation, Chem. Phys. Lett., 1999, 314, 291-299.

86 T. Yanai, D. P. Tew, and N. C. Handy, A new hybrid exchange-correlation functional using the Coulombattenuating method (CAM-B3LYP), Chem. Phys. Lett., 2004, 393, 51-57.

87 M. A. Rohrdanz and J. M. Herbert, Simultaneous benchmarking of ground- and excited-state properties with long-range-corrected density functional theory, J. Chem. Phys., 2008, 129, 034107:1-9.

${ }^{88}$ R. M. Richard and J. M. Herbert, Time-dependent density-functional description of the ${ }^{1} \mathrm{~L}_{a}$ state in polycyclic aromatic hydrocarbons: Charge-transfer character in disguise?, J. Chem. Theory Comput., 2011, 7, 12961306.

${ }^{89}$ L. D. Jacobson and J. M. Herbert, A one-electron model for the aqueous electron that includes many-body electron-water polarization: Bulk equilibrium structure, vertical electron binding energy, and optical absorption spectrum, J. Chem. Phys., 2010, 133, 154506:1-19.

90 S. Laricchia, E. Fabiano, L. Constantin, and F. D. Sala, Generalized gradient approximations of the noninteracting kinetic energy from the semiclassical atom theory: Rationalization of the accuracy of the frozen density embedding theory for nonbonded interactions, J. Chem. Theory Comput., 2011, 7, 2439-2451.

91 F. H. Stillinger, Energy Landscapes, Inherent Structures, and Condensed-Matter Phenomena, Princeton University Press, Princeton, NJ, 2016.

92 A. D. Laurent and D. Jacquemin, TD-DFT benchmarks: A review, Int. J. Quantum Chem., 2013, 113, 2019-2039.

93 A. Lange and J. M. Herbert, Simple methods to reduce charge-transfer contamination in time-dependent densityfunctional calculations of clusters and liquids, J. Chem. 
Theory Comput., 2007, 3, 1680-1690.

94 A. W. Lange, M. A. Rohrdanz, and J. M. Herbert, Charge-transfer excited states in a $\pi$-stacked adenine dimer, as predicted using long-range-corrected timedependent density functional theory, J. Phys. Chem. B, 2008, 112, 6304-6308, Erratum: ibid. 112, 7345 (2008).

95 J. M. Herbert, X. Zhang, A. F. Morrison, and J. Liu, Beyond time-dependent density functional theory using only single excitations: Methods for computational studies of excited states in complex systems, Acc. Chem. Res., 2016, 49, 931-941.

96 R. J. Magyar and S. Tretiak, Dependence of spurious charge-transfer excited states on orbital exchange in TDDFT: Large molecules and clusters, J. Chem. Theory Comput., 2007, 3, 976-987.

97 L. D. Jacobson and J. M. Herbert, Theoretical characterization of four distinct isomer types in hydratedelectron clusters, and proposed assignments for photoelectron spectra of water cluster anions, J. Am. Chem. Soc., 2011, 133, 19889-19899.

98 F. Uhlig, J. M. Herbert, M. P. Coons, and P. Jungwirth, Optical spectroscopy of the bulk and interfacial hydrated electron from ab initio calculations, J. Phys. Chem. A, 2014, 118, 7507-7515.

99 L. Turi and A. Madarász, Comment on "Does the hydrated electron occupy a cavity?", Science, 2011, 331, 1387.

100 L. D. Jacobson and J. M. Herbert, Comment on "Does the hydrated electron occupy a cavity?", Science, 2011, 331, 1387.

101 J. M. Herbert and L. D. Jacobson, Structure of the aqueous electron: Assessment of one-electron pseudopotential models in comparison to experimental data and timedependent density functional theory, J. Phys. Chem. A, 2011, 115, 14470-14483. 
Table of Contents Graphic

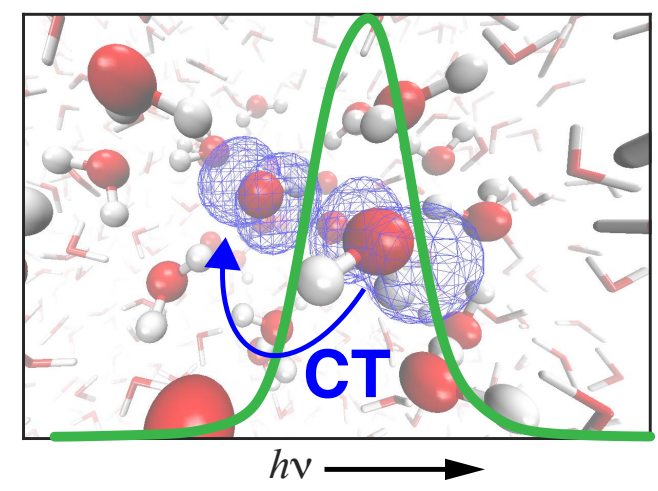

